\title{
ERROR ESTIMATES FOR THE FINITE ELEMENT APPROXIMATION OF A SEMILINEAR ELLIPTIC CONTROL PROBLEM WITH STATE CONSTRAINTS AND FINITE DIMENSIONAL CONTROL SPACE
}

\author{
Pedro Merino $^{1}$, Fredi TrÖltzsch $^{2}$ And Boris Vexler ${ }^{2}$
}

\begin{abstract}
The finite element approximation of optimal control problems for semilinear elliptic partial differential equation is considered, where the control belongs to a finite-dimensional set and state constraints are given in finitely many points of the domain. Under the standard linear independency condition on the active gradients and a strong second-order sufficient optimality condition, optimal error estimates are derived for locally optimal controls.
\end{abstract}

Mathematics Subject Classification. 49J20, 35B37.

Received April 19, 2008. Revised July 16, 2009.

Published online December 16, 2009.

\section{INTRODUCTION}

In this paper, we derive error estimates for a finite element based approximation of a semilinear elliptic optimal control problem with control out of a finite-dimensional set and constraints on the state which are required only in finitely many points of the spatial domain. This type of problems is interesting for several reasons. Finite-dimensional controls appear in many real applications of optimal control theory, because it is rather difficult to practically implement control functions that can vary arbitrarily in space. We refer also to [12], where examples of problems with practical importance are mentioned. If pointwise state constraints are given with finite-dimensional control space, then they are often active in finitely many points only. Therefore, finitely many pointwise constraints turn out to be interesting for numerical computations, if the location of active points has been detected.

Our problem is equivalent to a nonlinear programming problem in a finite-dimensional space. Therefore, the existence of optimal controls, first- or second-order optimality conditions are more or less standard results since they follow from classical results of mathematical programming after transforming the control problem into a finite-dimensional one. We refer to [12], where optimality conditions for semilinear control problems with finite-dimensional control space are discussed with pointwise state constraints given in the whole spatial domain.

The question of error estimates, however, is still interesting. Numerical computations indicate that the numerical approximation of the optimal controls and states is of the order $h^{2}$, where $h>0$ is the mesh size

\footnotetext{
Keywords and phrases. Finite element approximation, optimal control problem, finitely many pointwise state constraints.

1 Department of Mathematics, EPN Quito, Ecuador.

2 Institut für Mathematik, TU Berlin, Germany. troeltz@math.TU-Berlin.DE
} 
of the finite element scheme. We can show that the order of the error is equal to $h^{2}|\log h|$, which corresponds to the error estimate for the finite-element approximation of the state in $L^{\infty}$-norm. This is quite clear intuitively, since the control space is finite-dimensional and the state constraints are posed pointwise in a finite number of points. Then, no interpolation error of the optimal control occurs that in the case of control functions limits the order of convergence to $h$ for a cellwise constant approximation of the control variable, see, e.g., [3], and to $h^{3 / 2}$ for a piecewise linear approximation, see, e.g., $[9,30]$.

In [4], an optimal control problem of sound is considered, where the controls are chosen to be finite Dirac delta measures. If in addition, finitely many pointwise state constraints are imposed in points inside the domain, that do not coincide with the points where the Dirac delta measures are concentrated, then the problem can be also written as a nonlinear programming problem in finite dimensions and the results presented in our paper can also be applied. The error estimate for the finite element approximation obtained in [4] for the linear state equation is the same as ours. Therefore, under natural assumptions we would arrive at the error estimate of order $h^{2}|\log (h)|$ for the optimal point controls.

For pointwise state constraints in the whole domain, we know only a few results. In Casas [8], the convergence of finite element approximations to optimal control problems for semilinear elliptic equations with finitely many state constraints is proven for piecewise constant approximations of the control function. This result was extended by Casas and Mateos [10] to boundary control problems.

Error estimates were derived by Deckelnick and Hinze [13] and Meyer [24], who consider linear-quadratic problems with pointwise state constraints and obtain the order $h^{1-\varepsilon}$ for domains of dimension $d=2$ and $h^{1 / 2-\varepsilon}$ for $d=3$, under the assumption that the control function is not discretized. Recently, Deckelnick and Hinze [14] estimated the error by $h|\log h|$ for $d=2$ and $h^{1 / 2}$ for $d=3$ with piecewise constant approximation of the control.

Extending $L^{\infty}$-error estimates from [27] for linear elliptic equations to the semilinear case in two dimensions, we show that the order is precisely $h^{2}|\log h|$. At first glance, this is surprising since the Lagrange multipliers associated with the (finitely many) pointwise state constraints are regular Borel measures, which appear in the adjoint equation of the control system. It is the finite-dimensional nature of our problem that explains the high order of the error. We point out that the method presented here can be applied to three dimensional cases under the condition that an $L^{\infty}$ norm estimate for the state equations is available. In that case, the expected error is the same as the uniform norm estimate for the state equation.

This problem of error estimates leads to investigating a perturbed nonlinear programming problem, where the perturbation parameter is the mesh size $h$ - seemingly a standard problem of perturbation analysis. However, we were not able to find approximation results in the literature, which can be applied directly to our problem due to the difference in the assumptions on the perturbation parameter.

The paper is organized as follows: in Section 2 we define the optimal control problem (OCP), introduce the notation and state the assumptions. In Section 3, the finite element discretization is introduced and error estimates for the partial differential equations are derived. Moreover, this section is devoted to estimating the error for the solutions of the optimal control problem. Numerical examples are presented in Section 4, and Section 5 briefly sketches the error analysis for the perturbed nonlinear programming problem.

\section{Optimal CONTROL PROBlem AND OPtimality CONDITIONS}

\subsection{Definition of the problem and main assumptions}

We consider the following optimal control problem:

$(\mathrm{OCP})$

$$
\left\{\begin{array}{l}
\min _{u \in \mathcal{U}_{a d}} J\left(y_{u}, u\right)=\int_{\Omega} L\left(x, y_{u}(x), u\right) \mathrm{d} x \\
\text { subject to } \\
g_{i}\left(y_{u}\left(x_{i}\right)\right)=0, \quad \text { for all } i=1, \ldots, k, \\
g_{i}\left(y_{u}\left(x_{i}\right)\right) \leq 0, \quad \text { for all } i=k+1, \ldots, \ell,
\end{array}\right.
$$


where $y_{u}$ is the solution to the state equation

$$
\begin{aligned}
& A y(x)+d(x, y(x), u)=0 \text { in } \Omega \\
& y(x)=0 \text { on } \Gamma
\end{aligned}
$$

and $\mathcal{U}_{a d}$ is the set of box constraints defined by $\mathcal{U}_{a d}=\left\{u \in \mathbb{R}^{m}: u_{a} \leq u \leq u_{b}\right\}$ with given vectors $u_{a} \leq u_{b}$ of $\mathbb{R}^{m}$. We assume $\ell \geq 1$ and set $k=0$, if only inequality constraints are given and $k=\ell$, if only equality constraints are given.

The set $\Omega$ is a convex bounded open set in $\mathbb{R}^{n}, n=2$, with boundary $\Gamma$. For simplicity, we assume that $\Gamma$ is polygonal. The extension of some of our results to curved boundaries is possible along the lines of Arada et al. [3]. Moreover, functions $L, d: \Omega \times \mathbb{R}^{m+1} \rightarrow \mathbb{R}$, and $g_{i}: \mathbb{R} \mapsto \mathbb{R}$ of class $C^{2,1}$ are given together with points $x_{i} \in \Omega, i=1 \ldots \ell$. Notice that these points are not located at the boundary of $\Omega$.

The operator $A$ is a symmetric uniformly elliptic differential operator of the form

$$
A y(x)=-\sum_{i, j=1}^{n} \partial_{j}\left(a_{i j}(x) \partial_{i} y(x)\right)
$$

with coefficients $a_{i j} \in C^{1+\alpha}(\Omega), 0<\alpha<1$. The control $u \in \mathbb{R}^{m}$ is allowed to occur nonlinearly in the state equation, since the controls are vectors. For control functions, the proof of existence would be a delicate issue.

Notation. If not stated otherwise, we consider our vectors as column vectors. By $|\cdot|$, the Euclidean norm of vectors in spaces $\mathbb{R}^{n}$ is denoted, while $\|\cdot\|$ stands for the norm of matrices (defined by the Euclidean norm of the vector of all entries). The open ball around $u$ in $\mathbb{R}^{m}$ with radius $\rho$ is denoted by $B(u, \rho)$. If $f: \mathbb{R}^{n} \rightarrow \mathbb{R}$ is a twice differentiable function, then $f^{\prime}$ is its derivative and $\nabla f$ is the associated gradient, i.e. the representation of $f^{\prime}$ by a column vector. Moreover, $f^{\prime \prime}(z)$ denotes the Hessian matrix of $f$ at the vector $z$. We denote by $c$ a generic constant, while $C$ is a constant appearing in all estimates of our theorems; it is the maximum of all associated constants.

\section{Main assumptions}

Assumption 2.1 (Carathéodory type assumptions). For each fixed $x \in \Omega$, the functions $L=L(x, y, u)$ and $d=d(x, y, u)$ are of class $C^{2}$ with respect to $(y, u)$. For all fixed $(y, u)$ or fixed $y$, respectively, they are Hölder continuous with respect to the variable $x \in \Omega$.

Assumption 2.2 (Monotonicity). For all $x \in \Omega$, all $u \in \mathcal{U}_{\text {ad }}$ and $y \in \mathbb{R}$, it holds that

$$
\frac{\partial d}{\partial y}(x, y, u) \geq 0 \text {. }
$$

Assumption 2.3 (Boundedness and Lipschitz properties). There are a constant $C$ and, for all $M>0$, a constant $C_{L}(M)>0$ such that the estimates

$$
\begin{aligned}
|d(x, 0,0)|+\left|d^{\prime}(x, 0,0)\right|+\left\|d^{\prime \prime}(x, 0,0)\right\| & \leq C \\
\left\|d^{\prime \prime}\left(x, y_{1}, u_{1}\right)-d^{\prime \prime}\left(x, y_{2}, u_{2}\right)\right\|+\left\|g_{j}^{\prime \prime}\left(y_{1}\right)-g_{j}^{\prime \prime}\left(y_{2}\right)\right\| & \leq C_{L}(M)\left(\left|y_{1}-y_{2}\right|+\left|u_{1}-u_{2}\right|\right)
\end{aligned}
$$

hold for all $x \in \Omega$, all $u_{i} \in \mathcal{U}_{\text {ad }}$ and all $\left|y_{i}\right| \leq M, i=1,2, j=1, \ldots, \ell$. Here, $d^{\prime}$ and $d^{\prime \prime}$ denote the derivative and the Hessian matrix of $d(x, y, u)$ with respect to $(y, u)$, respectively.

The function $L$ is assumed to satisfy Assumption 2.3 accordingly.

Lemma 2.1. Under Assumptions 2.1-2.3, to each $u \in \mathcal{U}_{\text {ad }}$ there exists a unique solution $y_{u} \in H_{0}^{1}(\Omega) \cap C(\bar{\Omega})$. There is a constant $C$ such that $\left\|y_{u}\right\|_{L^{\infty}(\Omega)} \leq C$ is satisfied for all $u \in \mathcal{U}_{a d}$. If $\Omega$ is convex or of class $C^{2}$, then $y_{u}$ belongs to $H^{2}(\Omega)$. 
The first part of this result and the uniform boundedness are standard, we refer to Casas [7]. The $H^{2}$-regularity follows from results by Grisvard [18] on linear equations after taking the nonlinear terms with $d\left(\cdot, y_{u}, u\right) \in L^{\infty}(\Omega)$ to the right-hand side of the equation.

The state $y$ that is associated to the control $u$ by the PDE in (OCP), is denoted by $y_{u}$. We denote the mapping $u \mapsto y_{u}$ by $S: \mathbb{R}^{m} \rightarrow H_{0}^{1}(\Omega) \cap C(\bar{\Omega})$, i.e. $y_{u}=S(u) ; S$ is our control-to-state mapping.

Lemma 2.2. Under Assumptions 2.1-2.3, the control-to-state mapping $S: \mathbb{R}^{m} \rightarrow H_{0}^{1}(\Omega) \cap C(\bar{\Omega})$ is twice continuously Fréchet-differentiable. For arbitrary elements $u$ and $v$ of $\mathbb{R}^{m}$, the function $z_{v}(u):=S^{\prime}(u) v$ is given by $z_{v}(u)=z$, where $z$ is the unique solution to the problem

$$
\left\{\begin{aligned}
A z+\frac{\partial d}{\partial y}\left(x, y_{u}, u\right) z & =-\frac{\partial d}{\partial u}\left(x, y_{u}, u\right) v & & \text { in } \Omega \\
z & =0 & & \text { on } \Gamma,
\end{aligned}\right.
$$

and the inequality

$$
\left\|z_{v}(u)\right\|_{H^{1}(\Omega)}+\left\|z_{v}(u)\right\|_{C(\bar{\Omega})} \leq C|v|
$$

is satisfied with some constant $C$ that is independent of $u \in \mathcal{U}_{\text {ad }}$.

The function $z_{v_{1} v_{2}}(u):=S^{\prime \prime}(u)\left[v_{1}, v_{2}\right]$ is obtained by $z_{v_{1} v_{2}}=z$, where $z$ is the unique solution of

$$
\left\{\begin{aligned}
A z+\frac{\partial d}{\partial y}\left(x, y_{u}, u\right) z & =-\left(y_{v_{1}}^{\prime}, v_{1}^{\top}\right) d^{\prime \prime}\left(x, y_{u}, u\right)\left(y_{v_{2}}^{\prime}, v_{2}^{\top}\right)^{\top} \text { in } \Omega \\
z & =0
\end{aligned}\right.
$$

with $y_{v_{1}}^{\prime}=S^{\prime}(u)\left(v_{1}\right)$ and $y_{v_{2}}^{\prime}=S^{\prime}(u)\left(v_{2}\right)$.

For later use of the theory of nonlinear optimization in finite-dimensional spaces, we convert problem (OCP) into a finite-dimensional nonlinear programming problem. We introduce the reduced objective function $f: \mathbb{R}^{m} \rightarrow \mathbb{R}$ of class $C^{2,1}$ by

$$
f(u)=J\left(y_{u}, u\right)=J(S(u), u) .
$$

By our assumptions, in particular the Lipschitz properties of the second derivatives of the given nonlinear functions and the boundedness of $\mathcal{U}_{a d}$, it follows that $S^{\prime \prime}$ is Lipschitz on $\mathcal{U}_{a d}$, i.e. there exists a constant $C>0$ such that

$$
\left\|S^{\prime \prime}\left(u_{1}\right)\left[v_{1}, v_{2}\right]-S^{\prime \prime}\left(u_{2}\right)\left[v_{1}, v_{2}\right]\right\|_{H_{0}^{1}(\Omega) \cap C(\bar{\Omega})} \leq C\left|u_{1}-u_{2}\right|\left|v_{1}\right|\left|v_{2}\right| \quad \forall u_{i} \in \mathcal{U}_{a d}, v_{i} \in \mathbb{R}^{m}, i=1,2 .
$$

Moreover, we define the restriction function $G$ by

$$
G(u)=\left[g_{1}\left(y_{u}\left(x_{1}\right)\right), \ldots, g_{\ell}\left(y_{u}\left(x_{\ell}\right)\right)\right]^{\top}=\left[g_{1}\left(\delta_{1} S(u)\right), \ldots, g_{\ell}\left(\delta_{\ell} S(u)\right)\right]^{\top},
$$

where $\delta_{i}$ denotes the Dirac measure concentrated at the point $x_{i}, i=1, \ldots, \ell$. It shows that Dirac measures will naturally appear in the adjoint equation associated with (OCP).

By these definitions, (OCP) becomes equivalent to the finite-dimensional nonlinear programming problem (NP),

$$
\left\{\begin{array}{l}
\min f(u) \\
G_{i}(u)=0, \quad i=1, \ldots k, \\
G_{i}(u) \leq 0, \quad i=k+1, \ldots \ell, \\
u \in \mathcal{U}_{a d} .
\end{array}\right.
$$

Remark 2.1. This problem admits at least one optimal solution $\bar{u}$, if its admissible set is non-empty. This follows immediately by the Weierstraß theorem, since $f$ and $G$ are continuous and $\mathcal{U}_{a d}$ is compact. Therefore, by its equivalence to (NP), also (OCP) is solvable in this case. 


\section{The DisCRETIZED OPTIMAL CONTROL PROBLEM}

\subsection{Finite element scheme and discretized optimal control problem}

To discretize the optimal control problem, we introduce a finite element approximation of the state equation (2.1). We consider a family of meshes $\left\{\mathcal{T}_{h}\right\}_{h>0}$ consisting of triangles $T \in \mathcal{T}_{h}$ such that

$$
\cup_{T \in \mathcal{T}_{h}} T=\bar{\Omega}
$$

Notice that $\Omega$ was supposed to be polygonal for simplicity. For each triangle $T \in \mathcal{T}_{h}$, we introduce the diameter $\rho(T)$ of $T$, and the diameter $\sigma(T)$ of the largest circle contained in $T$. The mesh size $h$ is defined by $h=\max _{T \in \mathcal{T}_{h}} \rho(T)$. We impose the following regularity assumption on the grid:

Assumption 3.1. There exist two positive constants $\rho$ and $\sigma$ such that

$$
\frac{\rho(T)}{\sigma(T)} \leq \sigma \text { and } \frac{h}{\rho(T)} \leq \rho, \quad \forall T \in \mathcal{T}_{h},
$$

are fulfilled for all $h>0$.

Associated with the given triangulation $\mathcal{T}_{h}$, we introduce the set of piecewise linear and continuous functions

$$
Y_{h}=\left\{y_{h} \in C(\bar{\Omega}): y_{\left.h\right|_{T}} \in P_{1}(T) \forall T \in \mathcal{T}_{h}, y_{h}=0 \text { on } \Gamma\right\}
$$

where $P_{1}(T)$ denotes the set of affine real-valued functions defined on $T$. For convenience, we introduce the bilinear form

$$
a(y, \eta)=\int_{\Omega} \sum_{i, j=1}^{n} a_{i j}(x) \partial_{x_{i}} y \partial_{x_{j}} \eta \mathrm{d} x .
$$

The discrete state $y_{h}$ is defined as the (unique) element of $Y_{h}$ that satisfies the following finite element scheme associated with (2.1):

$$
a\left(y_{h}, \eta_{h}\right)+\int_{\Omega} d\left(x, y_{h}, u\right) \eta_{h} \mathrm{~d} x=0 \quad \forall \eta_{h} \in Y_{h}
$$

The following existence and approximation result was proven in [11] for distributed control functions in Lipschitz domains. It is obvious that it remains valid without changes for our case with control vectors. It shows that the discretized equation is well posed.

Theorem 3.1. Let Assumptions $2.1-2.3$ and 3.1 be satisfied. Then, for each $u \in \mathcal{U}_{a d}$, the equation (3.1) has a unique solution $y_{h, u} \in Y_{h}$. There exists a constant $C$ independent of $h$ and $u \in \mathcal{U}_{\text {ad }}$ such that, with $n=\operatorname{dim} \Omega$,

$$
\begin{aligned}
\left\|y_{u}-y_{h, u}\right\|_{L^{2}(\Omega)} & \leq C h^{2}\left\|y_{u}\right\|_{H^{2}(\Omega)} \\
\left\|y_{u}-y_{h, u}\right\|_{L^{\infty}(\Omega)} & \leq C h^{2-n / 2}\left\|y_{u}\right\|_{H^{2}(\Omega)} .
\end{aligned}
$$

To indicate the correspondence of $y_{h}$ to $u$, we will denote this solution also by $y_{h, u}$. The mapping $u \mapsto y_{h, u}$ is denoted by $S_{h}$. We will improve estimate (3.3) in an interior subdomain $\Omega_{0}$ containing all points $x_{i}$, where the state constraints are required. 
We approximate (OCP) by substituting the discretized equation (3.1) for the state equation and study the following (discretized) finite-dimensional control problem

$$
\left(\mathrm{OCP}_{h}\right) \quad\left\{\begin{aligned}
\min _{u \in \mathcal{U}_{a d}} J\left(y_{h, u}, u\right) & =\int_{\Omega} L\left(x, y_{h, u}(x), u\right) \mathrm{d} x \\
g_{i}\left(y_{h, u}\left(x_{i}\right)\right) & =0 \quad \text { for all } i=1, \ldots, k, \\
g_{i}\left(y_{h, u}\left(x_{i}\right)\right) \leq 0 & \text { for all } i=k+1, \ldots, \ell
\end{aligned}\right.
$$

Let us define

$$
f_{h}(u)=J\left(y_{h, u}, u\right), \quad G_{h}(u)=\left[g_{1}\left(y_{h, u}\left(x_{1}\right)\right), \ldots, g_{\ell}\left(y_{h, u}\left(x_{\ell}\right)\right)\right]^{\top}
$$

By these terms, we transform problem (3.4) to the finite-dimensional nonlinear programming problem

$$
\left\{\begin{array}{l}
\min f_{h}(u) \\
G_{h, i}(u)=0, \quad i=1, \ldots, k \\
G_{h, i}(u) \leq 0, \quad i=k+1, \ldots, \ell \\
u \in \mathcal{U}_{a d}
\end{array}\right.
$$

Remark 3.1. Notice that in the definition of the optimization problem $\left(\mathrm{NP}_{h}\right)$, neither the assignment $h \mapsto f_{h}(u)$ nor $h \mapsto G_{h, i}(u)$ are well defined mappings for a fixed $u$ because different meshes have the same mesh size. The values $f_{h}(u)$ or $G_{h, i}(u)$ depend on the mesh and they are not fixed by $h$.

\subsection{Improved $\boldsymbol{L}^{\infty}$-error estimate}

Let $\Omega_{0}$ with $\bar{\Omega}_{0} \subset \Omega$ be a subdomain of $\Omega$ that contains all given points $x_{i}, i=1, \ldots, \ell$. The main goal of this subsection is to estimate the $L^{\infty}$-norm of the difference $y_{u}-y_{h, u}$ in $\Omega_{0}$. We rely on an associated estimate for linear equations from [27] and on Theorem 3.1 by Casas and Mateos [11]. An estimate of the order $h^{2}|\log (h)|$ was derived in [27] for compact subdomains of polygonal domains. We also mention the related papers $[16,26]$, where the error estimate $h^{2}|\log (h)|$ was shown for domains with sufficiently smooth boundary. Another technique for proving local error estimates with respect to the $L^{\infty}$-norm is presented in [31,32]. Here, we extend the result of [27] to the semilinear case.

First, we show that the state $y_{u}$ belongs to $W^{2, \infty}\left(\Omega_{1}\right)$, where $\bar{\Omega}_{0} \subset \Omega_{1}$ and $\bar{\Omega}_{1} \subset \Omega$.

Lemma 3.1. Under Assumptions 2.1-2.3, the state $y_{u}$ belongs to $W^{2, \infty}\left(\Omega_{1}\right)$ for all $u \in \mathcal{U}_{a d}$.

Proof. We already know that $y_{u}$ is bounded and measurable, hence the same is true for $d\left(\cdot, y_{u}, u\right)$. Writing the state equation in the form $A y_{u}=-d\left(\cdot, y_{u}, u\right)$, we see that $y_{u}$ solves a linear elliptic equation with right-hand side in $L^{2}(\Omega)$. Since $\Omega$ is convex and bounded, $y_{u} \in H^{2}(\Omega)$ follows from Grisvard [18], hence $y_{u}$ is Hölder continuous, because $\Omega$ is two-dimensional. The smoothness and Lipschitz assumptions on $d$ ensure $d\left(\cdot, y_{u}, u\right) \in C^{0, \kappa}(\bar{\Omega})$ with some $\kappa \in(0,1)$. Therefore, $y_{u}$ solves a linear elliptic boundary value problem

$$
A y=f, \quad y_{\mid \Gamma}=0
$$

with Hölder continuous right-hand side $f$ and continuous boundary data. As a convex bounded domain, $\Omega$ satisfies the exterior sphere condition. In view of this, we can apply Theorem 6.13 by Gilbarg and Trudinger [17] and obtain $y_{u} \in C^{2, \alpha}(\Omega)$ with some $\alpha \in(0,1)$. This implies in particular $y_{u} \in C^{2, \alpha}\left(\bar{\Omega}_{1}\right)$, hence the statement of the lemma is true. 
Now we are going to derive a uniform error estimate of the order $h^{2}|\log h|$ in $\Omega_{0}$. The following estimate is known, e.g., from [27], for the linear case, $c f$. also [31,32]:

$$
\left\|y_{h, u}-y_{u}\right\|_{C\left(\Omega_{0}\right)} \leq C h^{2}|\log h|\left(\left\|y_{u}\right\|_{W^{2, \infty}\left(\Omega_{1}\right)}+\left\|y_{u}\right\|_{H^{2}(\Omega)}\right),
$$

where $\bar{\Omega}_{0} \subset \Omega_{1}$ and $\bar{\Omega}_{1} \subset \Omega$. We shall extend this estimate to the semilinear equation (2.1).

To establish our error estimate, we rely on the following stability result for linear elliptic equations from [27]:

Theorem 3.2. Let $\Omega_{1}$ be a subdomain of $\Omega$ with $\bar{\Omega}_{0} \subset \Omega_{1}$ and $\bar{\Omega}_{1} \subset \Omega$ and assume that $B \in\left(W^{1, \infty}(\Omega)\right)^{2 \times 2}$ and $\psi \in H_{0}^{1}(\Omega) \cap C(\bar{\Omega})$ are given. If $z_{h} \in Y_{h}$ is the solution of

$$
a\left(z_{h}, \eta_{h}\right)=\left(B \nabla \psi, \nabla \eta_{h}\right) \quad \forall \eta_{h} \in Y_{h},
$$

then there holds

$$
\left\|z_{h}\right\|_{L^{\infty}\left(\Omega_{0}\right)} \leq c\|B\|_{W^{1, \infty}(\Omega)}\left(|\log (h)|\|\psi\|_{L^{\infty}\left(\Omega_{1}\right)}+\|\psi\|_{L^{2}(\Omega)}+h\|\psi\|_{H^{1}(\Omega)}\right) .
$$

Our main error estimate for the state equation is expressed by the following result:

Theorem 3.3. Let $\Omega_{0}$ and $\Omega_{1}$ be defined as in Theorem 3.2. Then there exists a constant $C>0$, independent of $h$ and $u \in \mathcal{U}_{\text {ad }}$ such that it holds

$$
\left\|y_{u}-y_{h, u}\right\|_{L^{\infty}\left(\Omega_{0}\right)} \leq C\left(h^{2}|\log h|\left\|y_{u}\right\|_{W^{2, \infty}\left(\Omega_{1}\right)}+h^{2}\left\|y_{u}\right\|_{H^{2}(\Omega)}\right) .
$$

Proof. In the proof, we keep the control $u$ in (2.1) fixed. In view of this, we denote $y_{u}$ by $y$ and $y_{h, u}$ by $y_{h}$ to simplify the notation.

(i) The state $y$ is bounded, hence the $L^{\infty}$-estimate (3.3) implies uniform boundedness of $y_{h}$, independently of $h$. Therefore, we get from the Lipschitz continuity of $d$ with respect to $y$ and from the estimate (3.2)

$$
\left\|d(\cdot, y, u)-d\left(\cdot, y_{h}, u\right)\right\|_{L^{2}(\Omega)} \leq c\left\|y-y_{h}\right\|_{L^{2}(\Omega)} \leq c h^{2}\|y\|_{H^{2}(\Omega)} .
$$

We consider an intermediate solution $\tilde{y}_{h} \in Y_{h}$ determined by

$$
a\left(\tilde{y}_{h}, \eta_{h}\right)=-\left(d(\cdot, y, u), \eta_{h}\right) \quad \forall \eta_{h} \in Y_{h},
$$

where $(\cdot, \cdot)$ denotes the inner product of $L^{2}(\Omega)$. It is clear that $y$ satisfies the same equation, hence

$$
a\left(\tilde{y}_{h}, \eta_{h}\right)=a\left(y, \eta_{h}\right) \quad \forall \eta_{h} \in Y_{h} .
$$

Inserting the interpolate $i_{h} y$ of $y$, we obtain

$$
a\left(i_{h} y-\tilde{y}_{h}, \eta_{h}\right)=a\left(i_{h} y-y, \eta_{h}\right) \quad \forall \eta_{h} \in Y_{h} .
$$

Now we apply Theorem 3.2 to $\psi=i_{h} y-y$ and deduce

$$
\left\|i_{h} y-\tilde{y}_{h}\right\|_{L^{\infty}\left(\Omega_{0}\right)} \leq c\left(|\log h|\left\|i_{h} y-y\right\|_{L^{\infty}\left(\Omega_{1}\right)}+\left\|i_{h} y-y\right\|_{L^{2}(\Omega)}+h\left\|i_{h} y-y\right\|_{H^{1}(\Omega)}\right) .
$$

Due to known properties of the interpolation operator, $c f$. Brenner and Scott [6], we have

$$
\begin{aligned}
\left\|i_{h} y-y\right\|_{L^{2}(\Omega)}+h\left\|i_{h} y-y\right\|_{H^{1}(\Omega)} & \leq c h^{2}\|y\|_{H^{2}(\Omega)} \\
\left\|i_{h} y-y\right\|_{L^{\infty}\left(\Omega_{i}\right)}+h\left\|i_{h} y-y\right\|_{W^{1, \infty}\left(\Omega_{i}\right)} & \leq c h^{2}\|y\|_{W^{2, \infty}\left(\Omega_{i}\right)}, \quad i=0,1 .
\end{aligned}
$$


Using these interpolation estimates in (3.8), we arrive at

$$
\left\|y-\tilde{y}_{h}\right\|_{L^{\infty}\left(\Omega_{0}\right)} \leq c\left(h^{2}|\log h|\|y\|_{W^{2, \infty}\left(\Omega_{1}\right)}+h^{2}\|y\|_{H^{2}(\Omega)}\right) .
$$

(ii) It remains to estimate the error $v_{h}=\tilde{y}_{h}-y_{h} \in Y_{h}$. Subtracting the equations for $\tilde{y}_{h}$ and $y_{h}$, we find that $v_{h}$ solves

$$
a\left(v_{h}, \eta_{h}\right)=\left(d\left(\cdot, y_{h}, u\right)-d(\cdot, y, u), \eta_{h}\right) \quad \forall \eta_{h} \in Y_{h} .
$$

Now consider the function $v \in H_{0}^{1}(\Omega) \cap C(\bar{\Omega})$ defined by

$$
a(v, \eta)=\left(d\left(\cdot, y_{h}, u\right)-d(\cdot, y, u), \eta\right) \quad \forall \eta \in H_{0}^{1}(\Omega) .
$$

Obviously, $v_{h}$ is the discrete solution belonging to $v$. From standard estimates for linear elliptic equations and from inequality (3.7), it follows that

$$
\|v\|_{L^{\infty}(\Omega)}+\|v\|_{H^{1}(\Omega)} \leq c\left\|d(\cdot, y, u)-d\left(\cdot, y_{h}, u\right)\right\|_{L^{2}(\Omega)} \leq c h^{2}\|y\|_{H^{2}(\Omega)} .
$$

Now, we apply Theorem 3.2 to the linear equation

$$
a\left(v_{h}, \eta_{h}\right)=a\left(v, \eta_{h}\right) \quad \forall \eta_{h} \in Y_{h}
$$

and obtain

$$
\left\|v_{h}\right\|_{L^{\infty}\left(\Omega_{0}\right)} \leq c\left(|\log (h)|\|v\|_{L^{\infty}\left(\Omega_{1}\right)}+\|v\|_{L^{2}(\Omega)}+h\|v\|_{H^{1}(\Omega)}\right) \leq c|\log (h)| h^{2}\|y\|_{H^{2}(\Omega)} .
$$

In view of (3.9), the inequality $\left\|y-y_{h}\right\|_{L^{\infty}\left(\Omega_{0}\right)} \leq\left\|y-\tilde{y}_{h}\right\|_{L^{\infty}\left(\Omega_{0}\right)}+\left\|v_{h}\right\|_{L^{\infty}\left(\Omega_{0}\right)}$ yields the statement of the theorem.

\subsection{Application to the optimal control problem}

Let us first summarize the error estimates of the preceding subsection in the following lemma:

Lemma 3.2. Let $y_{u}$ and $y_{h, u}$ be the solutions of the equations (2.1) and (3.1), respectively. Let $z_{v}(u)$ solve (2.2) and $z_{v, h}(u)$ satisfy the corresponding discretized equation. Then there exists a constant $C>0$, independent of $h$, such that the estimates

$$
\begin{aligned}
\left\|y_{u}-y_{h, u}\right\|_{C\left(\bar{\Omega}_{0}\right)} & \leq C h^{2}|\log h| \\
\left\|z_{v}(u)-z_{v, h}(u)\right\|_{C\left(\bar{\Omega}_{0}\right)} & \leq C h^{2}|\log h||v|
\end{aligned}
$$

hold for all $u \in \mathcal{U}_{a d}, v \in \mathbb{R}^{m}$ and $h>0$.

Proof. Lemma 2.1 and the Lipschitz properties of $d$ ensure the existence of a bound $M>0$ with $\| d\left(\cdot, y_{u}\right.$, $u) \|_{L^{2}(\Omega)} \leq M$ for all $u \in \mathcal{U}_{a d}$. In view of this and by Lemma 3.1, $\left\|y_{u}\right\|_{H^{2}(\Omega)}$ and $\left\|y_{u}\right\|_{W^{2, \infty}\left(\Omega_{1}\right)}$ are uniformly bounded for all $u \in \mathcal{U}_{a d}$ and (3.10) is an immediate conclusion of Theorem 3.3. Estimate (3.11) follows from (3.10) as in [3], Proposition 7.1. Notice that, due to the boundedness of $\mathcal{U}_{a d}$, the functions $y_{u}$ and $y_{h, u}$ belong to a bounded set. This is used for the proof in [3].

Lemma 3.2 implies an estimate of $G-G_{h}$ in the $C^{2}$-norm:

Lemma 3.3. Under Assumptions 2.1-2.3 and 3.1, there is a constant $C>0$ such that

$$
\left|G(u)-G_{h}(w)\right|+\left\|G^{\prime}(u)-G_{h}^{\prime}(w)\right\|+\sum_{i=1}^{\ell}\left\|G_{i}^{\prime \prime}(u)-G_{h, i}^{\prime \prime}(w)\right\| \leq C\left(|u-w|+h^{2}|\log h|\right)
$$

holds for all $u, w$ in $\mathcal{U}_{a d}$. 
Proof. Let $c$ denote a generic constant. Taking into account that all $g_{i}$ are of class $C^{2,1}$, hence also the functions $G_{i}$. In view of Lemma 3.2 we have

$$
\begin{aligned}
\left|G_{i}(u)-G_{h, i}(w)\right| & =\left|g_{i}\left(y_{u}\left(x_{i}\right)\right)-g_{i}\left(y_{h, w}\left(x_{i}\right)\right)\right| \leq c\left\|y_{u}-y_{h, w}\right\|_{C\left(\bar{\Omega}_{0}\right)} \\
& \leq c\left\|y_{u}-y_{w}\right\|_{C\left(\bar{\Omega}_{0}\right)}+c\left\|y_{w}-y_{h, w}\right\|_{C\left(\bar{\Omega}_{0}\right)} \\
& \leq c|u-w|+c h^{2}|\log h| \quad \forall i=1, \ldots, \ell,
\end{aligned}
$$

where the last inequality is obtained since the mapping $u \mapsto y_{u}$ is Lipschitz continuous. For the derivative $G^{\prime}$, we argue similarly. Let $w$ be an arbitrary unit vector of $\mathbb{R}^{m}$. Then

$$
\begin{aligned}
\left|G_{i}^{\prime}(u) v-G_{h, i}^{\prime}(w) v\right| \leq & \left|G_{i}^{\prime}(u) v-G_{i}^{\prime}(w) v\right|+\left|G_{i}^{\prime}(w) v-G_{h, i}^{\prime}(w) v\right| \\
\leq & \left|g_{i}^{\prime}\left(y_{u}\left(x_{i}\right)\right)\left(S^{\prime}(u) v\right)\left(x_{i}\right)-g_{i}^{\prime}\left(y_{w}\left(x_{i}\right)\right)\left(S^{\prime}(w) v\right)\left(x_{i}\right)\right| \\
& +\left|g_{i}^{\prime}\left(y_{w}\left(x_{i}\right)\right)\left(S^{\prime}(w) v\right)\left(x_{i}\right)-g_{i}^{\prime}\left(y_{h, w}\left(x_{i}\right)\right)\left(S_{h}^{\prime}(w) v\right)\left(x_{i}\right)\right| \\
\leq & c\left\|g_{i}^{\prime}\left(y_{u}\right) z_{v}(u)-g_{i}^{\prime}\left(y_{w}\right) z_{v}(w)\right\|_{C\left(\bar{\Omega}_{0}\right)} \\
& +\left\|g_{i}^{\prime}\left(y_{w}\right) z_{v}(w)-g_{i}^{\prime}\left(y_{h, w}\right) z_{h, v}(w)\right\|_{C\left(\bar{\Omega}_{0}\right)},
\end{aligned}
$$

where $z_{v}(u)$ and $z_{v}(w)$ are defined as in Theorem 2.2 and $z_{h, v}(w)$ is the discrete state associated with $z_{v}(w)$. With a constant $C$ we obtain that

$$
\begin{aligned}
& \left\|y_{u}-y_{w}\right\|_{C\left(\bar{\Omega}_{0}\right)} \leq C|u-w|, \quad\left\|y_{w}-y_{h, w}\right\|_{C\left(\bar{\Omega}_{0}\right)} \leq C h^{2}|\log h| \\
& \left\|z_{v}(w)-z_{h, v}(w)\right\|_{C\left(\bar{\Omega}_{0}\right)} \leq C h^{2}|\log h||v|
\end{aligned}
$$

for all $u, w \in \mathcal{U}_{a d}$ and all $v \in \mathbb{R}^{m}$. The first inequality expresses the Lipschitz continuity of $u \mapsto y_{u}$, while the second and third one follow from Lemma 3.2. The estimates for $G$ and $G^{\prime}$ are deduced now immediately from the estimates above. For $G^{\prime \prime}$, the technique is analogous to the one for $G^{\prime}$, since $\left\|z_{v_{1} v_{2}}(w)-z_{h, v_{1} v_{2}}(w)\right\|_{C\left(\bar{\Omega}_{0}\right)} \leq$ $C h^{2}|\log h|\left|v_{1}\right|\left|v_{2}\right|$ follows from equation (2.2) that has the same structure as (2.4).

Lemma 3.4. Under the assumptions of Lemma 3.3, the estimate

$$
\left|f(u)-f_{h}(u)\right|+\left|f^{\prime}(u)-f_{h}^{\prime}(u)\right|+\left\|f^{\prime \prime}(u)-f_{h}^{\prime \prime}(u)\right\| \leq C h^{2} \quad \forall u \in \mathcal{U}_{a d}
$$

is satisfied with some constant $C$ not depending on $h$ and $u$.

Proof. The function $L$ is Lipschitz on bounded sets and, in view of Lemma 2.1 and (3.3), $\left\|y_{u}\right\|_{C(\bar{\Omega})}$ and $\left\|y_{h, u}\right\|_{C(\bar{\Omega})}$ are uniformly bounded for all $u \in \mathcal{U}_{a d}$ and all $h$. Therefore, we find

$$
\left|f_{h}(u)-f(u)\right| \leq \int_{\Omega}\left|L\left(x, y_{h, u}, u\right)-L\left(x, y_{u}, u\right)\right| \mathrm{d} x \leq c\left\|y_{h, u}-y_{u}\right\|_{L^{2}(\Omega)} \leq C h^{2}
$$

by (3.2). Similarly, we can estimate the second part

$$
\begin{aligned}
\left(f^{\prime}(u)-f_{h}^{\prime}(u)\right) v= & \int_{\Omega}\left(\frac{\partial L}{\partial y}\left(x, y_{u}, u\right) z_{v}(u)-\frac{\partial L}{\partial y}\left(x, y_{h, u}, u\right) z_{h, v}(u)\right) \mathrm{d} x \\
& +\int_{\Omega}\left(\frac{\partial L}{\partial u}(x, y, u) v-\frac{\partial L}{\partial u}\left(x, y_{h, u}, u\right) v\right) \mathrm{d} x
\end{aligned}
$$

by (3.2) and the associated $L^{2}$-version of (3.11),

$$
\left\|z_{v}(u)-z_{v, h}(u)\right\|_{L^{2}(\Omega)} \leq C h^{2}|v|
$$


for all unit vectors $v$. As in the last proof, the estimation of $\left\|f^{\prime \prime}(u)-f_{h}^{\prime \prime}(u)\right\|$ is analogous to that of $\left|f^{\prime}(u)-f_{h}^{\prime}(u)\right|$.

Let us now state the main result of our paper, an error estimate for optimal controls of the discretized optimal control problem $\left(\mathrm{OCP}_{h}\right)$. This estimate follows from our error analysis for nonlinear mathematical programming problems in Section 5 as a corollary of Theorem 5.3 applied to $(\mathrm{NP})$ and $\left(\mathrm{NP}_{h}\right)$. To perform our perturbation analysis, we also need the well known linear independence of active gradients condition. We refer for instance to [21] or [15].

For the next definition and later use, we extend the vector $G(u)$ to $\hat{G}(u) \in \mathbb{R}^{\ell+2 m}$ by including the box constraints defining $\mathcal{U}_{a d}$

$$
\begin{aligned}
& G_{\ell+i}(u)=u_{a, i}-u_{i}, \quad \text { for } i=1, \ldots, m \\
& G_{\ell+m+i}(u)=u_{i}-u_{b, i}, \quad \text { for } i=1, \ldots, m,
\end{aligned}
$$

and $\hat{G}_{i}(u):=G_{i}(u), i=1, \ldots, \ell+2 m$.

Definition 3.1. The index set $\mathcal{A}(\bar{u})$ of active constraints at $\bar{u}$ is defined by

$$
\mathcal{A}(\bar{u})=\left\{i \in\{1, \ldots, \ell+2 m\}: G_{i}(\bar{u})=0\right\} .
$$

We say that the linear independence constraint qualification (LICQ) holds at $\bar{u}$ if the vectors $\nabla G_{i}(\bar{u})$ of the set of active gradients $\left\{\nabla G_{i}(\bar{u}) \mid i \in \mathcal{A}(\bar{u})\right\}$ are linearly independent.

Theorem 3.4. Suppose that Assumptions 2.1-3.1 are fulfilled and let $\bar{u}$ be a locally optimal control of problem (OCP) satisfying the linear independence condition (LICQ) and the standard strong second-order condition (5.7). Then $\bar{u}$ is locally unique and there exists a sequence $\bar{u}_{h}$ of locally optimal controls of the corresponding FEM-approximated problem $\left(\mathrm{OCP}_{h}\right)$ and a constant $C>0$ independent of $h$ such that the estimate

$$
\left|\bar{u}-\bar{u}_{h}\right| \leq C h^{2}|\log h|
$$

is satisfied for all sufficiently small $h$.

Proof. The optimal control problems $(\mathrm{OCP})$ and $\left(\mathrm{OCP}_{h}\right)$ are equivalent to the nonlinear programming problems $(\mathrm{NP})$ and $\left(\mathrm{NP}_{h}\right)$, which are particular cases of the nonlinear programming problems $(\mathrm{P})$ and $\left(\mathrm{P}_{h}\right)$, which will be discussed in Section 5. Due to the previous error estimates, Assumption 5.1 is fulfilled with $\alpha(h)=h^{2}|\log h|$. Therefore, Theorem 5.3 is applicable to $(\mathrm{NP})$ and $\left(\mathrm{NP}_{h}\right)$ and Theorem 3.4 is a direct conclusion.

Remark 3.2. The same estimate holds true for the vectors of Lagrange multipliers $\bar{\nu}$ and $\bar{\nu}_{h}$ associated with $\bar{u}$ and $\bar{u}_{h}$, i.e. $\left|\bar{\nu}-\bar{\nu}_{h}\right| \leq C h^{2}|\log h|$. These multipliers are associated with the state and control constraints, cf. Theorem 5.3.

\section{Numerical EXAMPLES}

In this section we present numerical test examples for which we confirm error estimates of an order close to $\alpha(h)=h^{2}$. This does not contradict our theory, since the $|\log h|$-term can hardly be detected numerically. Notice that $h^{2} \log h \leq c h^{2-\varepsilon}$ holds for all $\varepsilon>0$ and all sufficiently small $h>0$.

In the test runs, regular triangulations of the domain were generated by the Matlab pde-toolbox command initmesh and successively refined using the command refmesh. The discretized nonlinear optimal control problems were solved by an SQP method where the associated nonlinear programming problems were treated on using the $O O Q P^{3}$ solver. The right-hand side of the state equation of the examples was assembled in an exact fashion, i.e. we do not approximate the integral term involving functions $e_{i}$, because our theory does not include approximation errors for integrals. Therefore, the corresponding right-hand side variational terms

\footnotetext{
${ }^{3}$ OOQP: Object Oriented Software for Quadratic Programming, Mike Gertz, Steve Wright. http://pages.cs.wisc.edu/ swright/ooqp/. OOQP is copyrighted to the University of Chicago.
} 
$\int_{\Omega} e_{i} \eta_{k} \mathrm{~d} x$, where $\eta_{k}, k=1, \ldots, n_{h}$ are the piecewise linear basis functions of $Y_{h}$, were assembled computing the corresponding primitive over its limits in each triangle. The experimental error of convergence is computed by

$$
E O C=\frac{\log \left(\left|u-u_{h_{1}}\right|\right)-\log \left(\left|u-u_{h_{2}}\right|\right)}{\log \left(h_{1}\right)-\log \left(h_{2}\right)}
$$

for two consecutive mesh sizes $h_{1}$ and $h_{2}$.

Example 4.1. This is a slight modification of an example in [25], where the optimal state is active in one single point. The semilinear state equation is considered in the open unit disk $\Omega=B(0,1)$ :

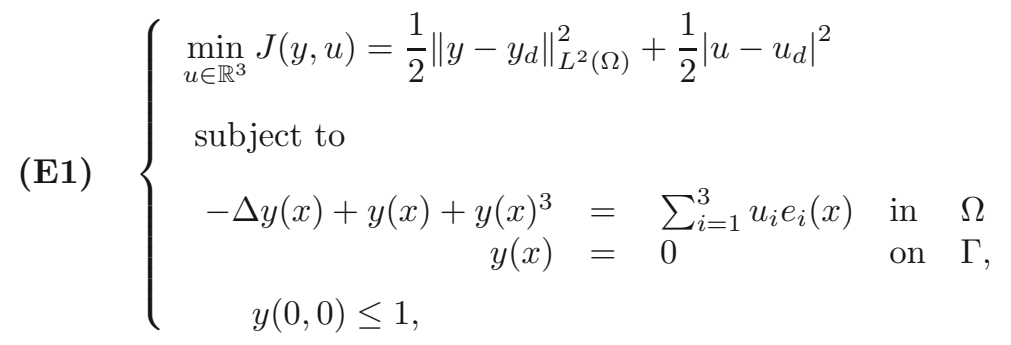

where $e_{1}(x)=1, e_{2}(x)=x_{1}^{2}+x_{2}^{2}, e_{3}(x)=\left(1-x_{1}^{2}-x_{2}^{2}\right)^{3}$. In this case, we construct the optimal functions

$$
\bar{y}=1-\left(x_{1}^{2}+x_{2}^{2}\right) \quad \text { and } \quad \bar{u}=[5,-1,1]^{\top} .
$$

The active set consists of one single point $x=(0,0)$. The Lagrange multiplier $\mu=\delta_{0}$ satisfies the complementarity condition, where $\delta_{0}$ is the Dirac measure concentrated at the origin. If we define $\bar{\varphi}=-\frac{1}{2 \pi} \log |x|$, then we have that

$$
-\Delta \bar{\varphi}+\bar{\varphi}+3 \bar{y}^{2} \bar{\varphi}=\delta_{0}-\frac{1}{2 \pi} \log |x|\left(1+3\left(1-|x|^{2}\right)^{2}\right)
$$

and it is easy to confirm that the right-hand side of this identity is equal to $\bar{y}-y_{d}+\bar{\mu}$, if we set

$$
y_{d}=1-|x|^{2}+\frac{1}{2 \pi} \log |x|\left(1+3\left(1-|x|^{2}\right)^{2}\right)
$$

The gradient equation for $\bar{u}$ is satisfied, if we define

$$
u_{d}=[5,-1,1]^{\top}+\left[\frac{1}{4}, \frac{1}{16}, \frac{25}{192}\right]^{\top} .
$$

Figure 1 shows the table with the numerically computed rate of convergence and the plot of the error for different mesh sizes compared with $h^{2}$ in logarithmic scale. The numerically computed optimal state and the desired state are shown in Figure 2. Notice that the state is active in the point $(0,0)$. In Figure 3, the computed Lagrange multiplier and the computed adjoint state are presented. The multiplier has a peak shape concentrated at $(0,0)$. This reflects the approximation of the Dirac measure $\delta_{0}$, which is the exact Lagrange multiplier.

Example 4.2. Here, the state equation contains a nonlinearity of the type $y^{2}$. Since $y^{2}$ is not monotone, it is replaced by the monotone function $y|y|$ for formal reasons. In the numerical computations, this does not matter, because the states turned out to be non-negative. We require that the optimal state $\bar{y}$ is active at five 


\begin{tabular}{|l|l|l|}
\hline$h$ & $\left|\bar{u}-\bar{u}_{h}\right|$ & $E O C$ \\
\hline 0.08192427 & 0.96438176 & \\
0.05016470 & 0.19273740 & 3.28 \\
0.02508235 & 0.05252443 & 1.88 \\
0.01263883 & 0.01450992 & 1.86 \\
0.00633700 & 0.00405185 & 1.85 \\
0.00317013 & 0.00119422 & 1.76 \\
\hline
\end{tabular}

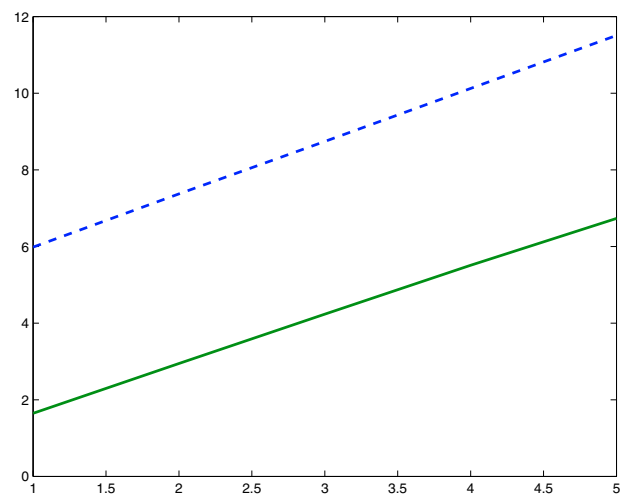

Figure 1. Example 4.1: table of error and plot $-\log (h)$ versus $-\log \left(\left|\bar{u}-\bar{u}_{h}\right|\right)$ (solid line) compared with $-2 \log (h)$ (dashed line).
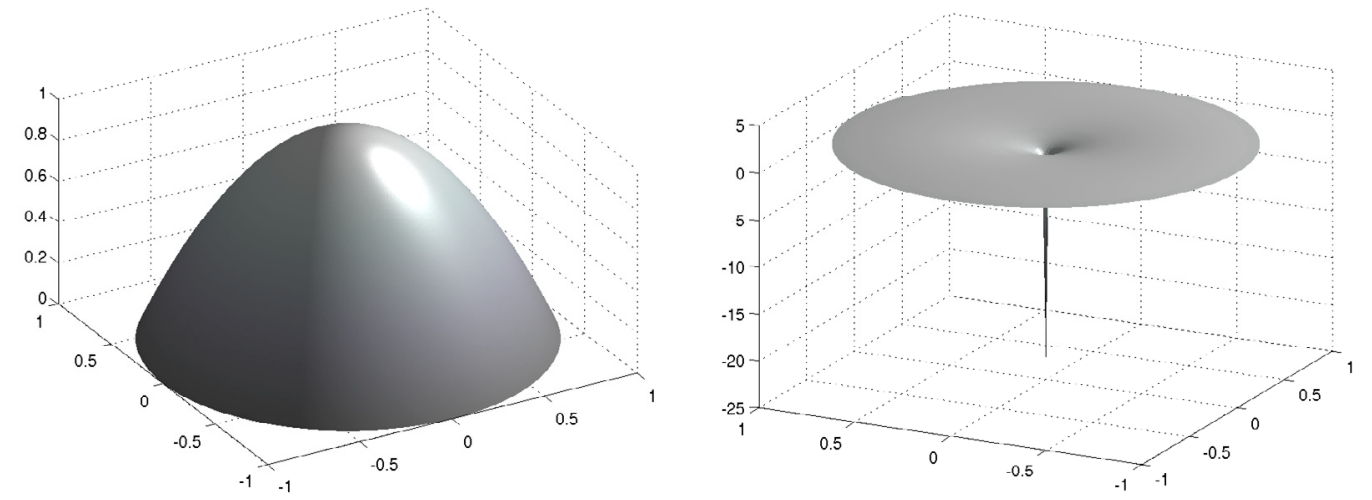

FIGURE 2. Example 4.1; numerically computed optimal state $\bar{y}_{h}$ and desired state $y_{d}$ for $h \approx 0.0006$.
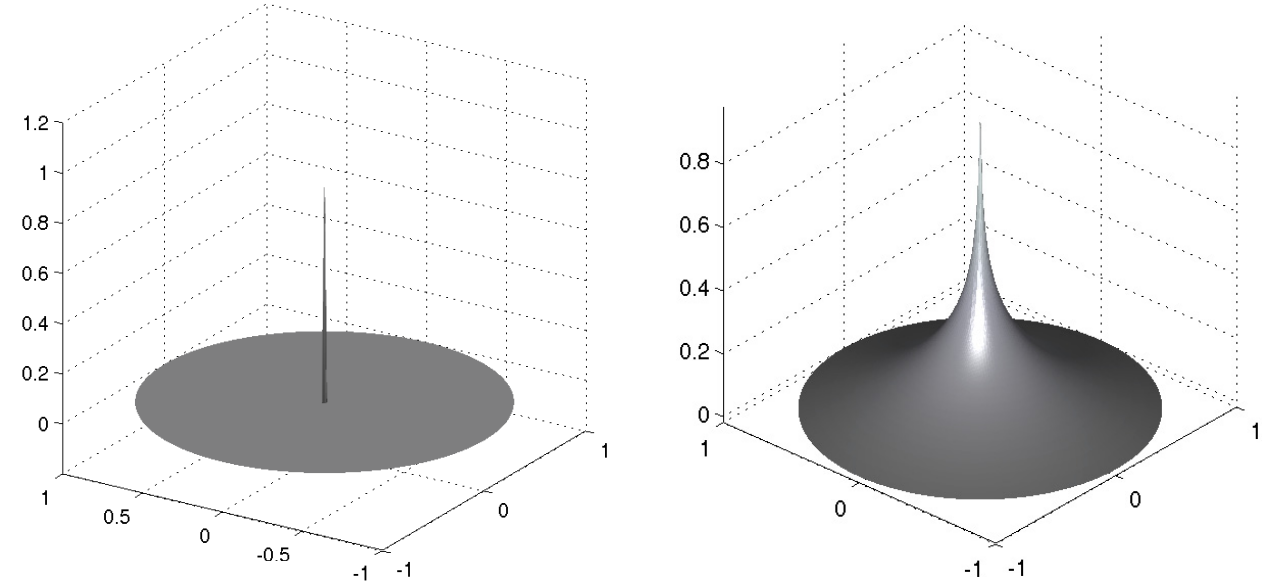

FiguRE 3. Example 4.1; numerically computed Lagrange multiplier $\bar{\mu}_{h}$ and adjoint state $\bar{\varphi}_{h}$ for $h \approx 0.0006$. 
given points inside the domain $\Omega=(-1,1) \times(-1,1)$. The problem reads

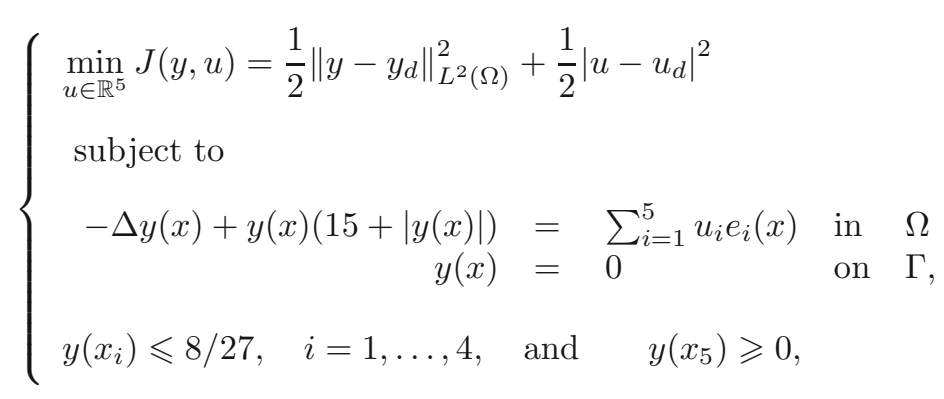

with

$$
x_{1}=\left(\frac{1}{\sqrt{3}}, \frac{1}{\sqrt{3}}\right), x_{2}=\left(-\frac{1}{\sqrt{3}}, \frac{1}{\sqrt{3}}\right), x_{3}=\left(\frac{1}{\sqrt{3}},-\frac{1}{\sqrt{3}}\right), x_{4}=\left(-\frac{1}{\sqrt{3}},-\frac{1}{\sqrt{3}}\right), x_{5}=(0,0),
$$

and

We define

$$
\begin{aligned}
& e_{1}(x)=12 x_{1}^{2} x_{2}^{2}-2\left(x_{1}^{4}+x_{2}^{4}\right), \quad e_{2}(x)=x_{1}^{2}+x_{2}^{2}, \quad e_{3}(x)=1, \\
& e_{4}(x)=\left(x_{1}^{2}-1\right)\left(x_{2}^{2}-1\right)\left(x_{1}^{2}+x_{2}^{2}\right), \quad e_{5}(x)=\left(x_{1}^{2}-1\right)^{2}\left(x_{2}^{2}-1\right)^{2}\left(x_{1}^{2}+x_{2}^{2}\right)^{2} .
\end{aligned}
$$

$$
\bar{y}=\left(x_{1}^{2}-1\right)\left(x_{2}^{2}-1\right)\left(x_{1}^{2}+x_{2}^{2}\right), \quad \bar{u}=[-2,16,-4,15,1]^{\top}
$$

so that $\bar{y}$ is the state associated with $\bar{u}$. Moreover, we set $y_{d}:=\bar{y}, u_{d}:=\bar{u}$ so that $\bar{y}$ and $\bar{u}$ are the optimal state and the optimal control, respectively. Consider the set

$$
\left\{\left(\frac{1}{\sqrt{3}}, \frac{1}{\sqrt{3}}\right),\left(-\frac{1}{\sqrt{3}}, \frac{1}{\sqrt{3}}\right),\left(\frac{1}{\sqrt{3}},-\frac{1}{\sqrt{3}}\right),\left(-\frac{1}{\sqrt{3}},-\frac{1}{\sqrt{3}}\right),(0,0)\right\}
$$

where the function $\bar{y}$ attains its maximal value $\frac{8}{27}$ in the first four points, while $\bar{y}$ reaches its minimum value 0 at the point $(0,0)$. In view of this choice, $\bar{y}$ is active at the five points, if the state constraints are chosen as above. Therefore, $\bar{y}$ and $\bar{u}$ are also the solution for the state-constrained problem. The gradient equation of the optimality system is

$$
\bar{u}-u_{d}+\left[\int_{\Omega} \bar{\varphi} e_{1} \mathrm{~d} x, \ldots, \int_{\Omega} \bar{\varphi} e_{5} \mathrm{~d} x\right]^{\top}=0
$$

In the computations, we chose an initial mesh that contains the five points where the state is active. Then all refined meshes automatically contain these five points. The computed rates for the error are listed in Figure 4 together with the plot of the approximation error and $h^{2}$ in logarithmic scale. Figure 5 shows the numerically computed optimal state, and the Lagrange multiplier is represented in Figure 6. It exhibits peaks in the points where the state is active, this is what we expected from the right-hand side in the adjoint equation (5.5) where Dirac measures are concentrated at the active points.

\section{ERror estimates For a PERTURBED NONLINEAR PROGRAMMing PROBLEM}

\subsection{The programming problem and its perturbation}

In the preceding section, we have transformed our elliptic optimal control problem into the nonlinear programming problem (NP). Associated with (NP), a discretized control problem was constructed that is equivalent 


\begin{tabular}{|l|l|l|}
\hline$h$ & $\left|\bar{u}-\bar{u}_{h}\right|$ & EOC \\
\hline 0.05700502 & 0.88041580 & - \\
0.02850251 & 0.25148686 & 1.81 \\
0.01425125 & 0.06332499 & 1.99 \\
0.00712562 & 0.01560647 & 2.02 \\
0.00356281 & 0.00382244 & 2.03 \\
\hline
\end{tabular}

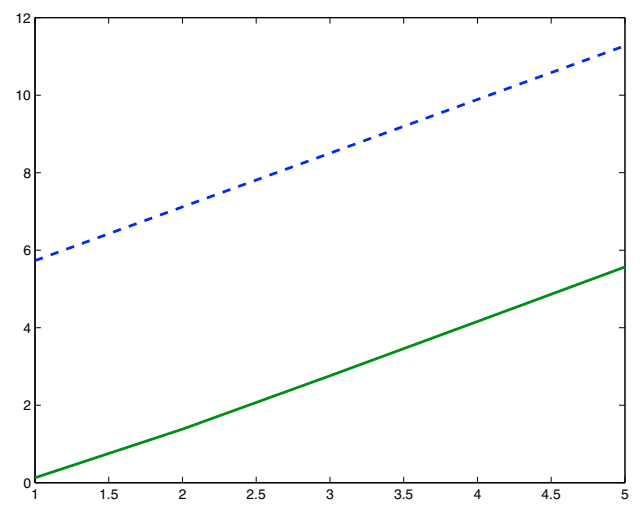

Figure 4. Example 4.2: table of error and plot $-\log (h)$ versus $-\log \left(\left|\bar{u}-\bar{u}_{h}\right|\right)$ (solid line) compared with $-2 \log (h)$ (dashed line).
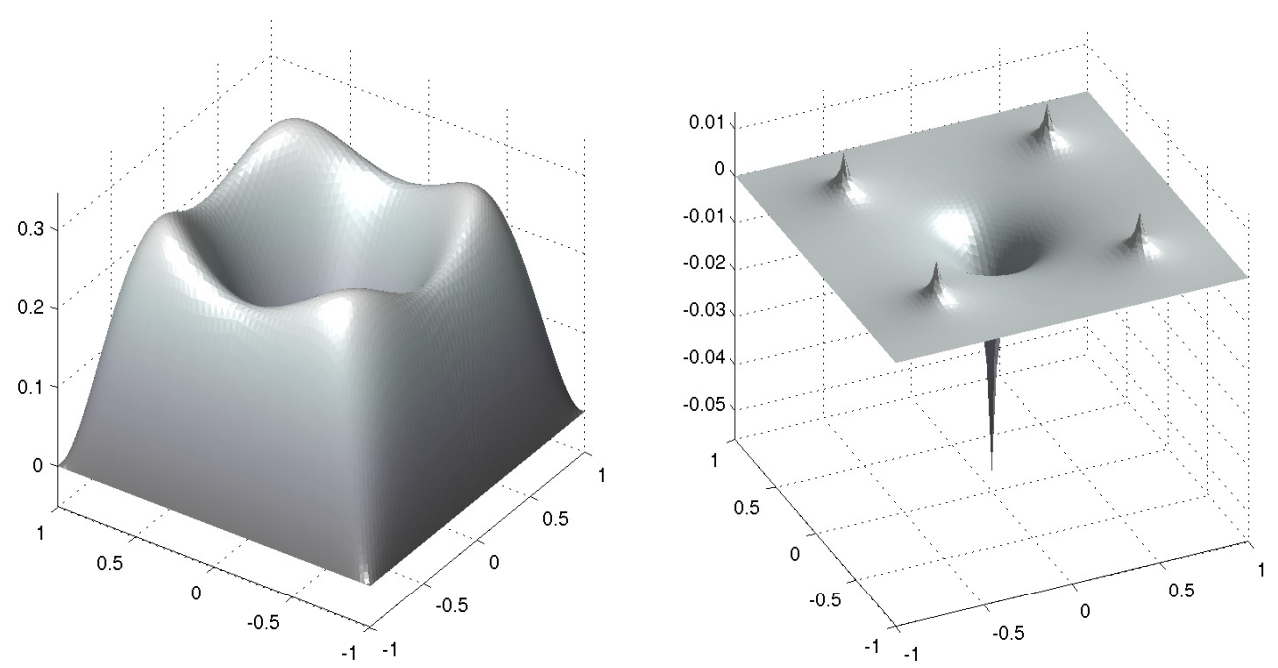

Figure 5. Example 4.2: numerically computed optimal state, and adjoint state at $h \approx 0.005$.

to the programming problem $\left(\mathrm{NP}_{h}\right)$. We are interested in estimating the difference between local solutions of $(\mathrm{NP})$ and associated solutions of $\left(\mathrm{NP}_{h}\right)$.

This question leads to the sensitivity analysis of nonlinear programming problems with respect to perturbations. Although this issue was the subject of various papers, in most of the references we found, the dependence of the given functions with respect to $h$ is assumed to be Lipschitz or even smoother, because Lipschitz stability or differentiability of the solutions with respect to $h$ was the main interest.

In our case, $h$ is only a mesh parameter, and hence we are faced with the difficulties stated in Remark 3.1. The only property we have at our disposal is the continuity of the data at $h=0$ with some associated rate. Results contained in Bonnans and Shapiro [5], Klatte und Kummer [20], Malanowski [22] or in many of the references cited therein, cannot be directly used, although their techniques need only a slight modification to be applied to our setting. Similarly, the results presented in Alt [2], Klatte [19] or Malanowski et al. [23], would need some adaptation and the verification of the assumptions stated therein. These papers only assume a rate of continuity with respect to $h$ at $h=0$. 

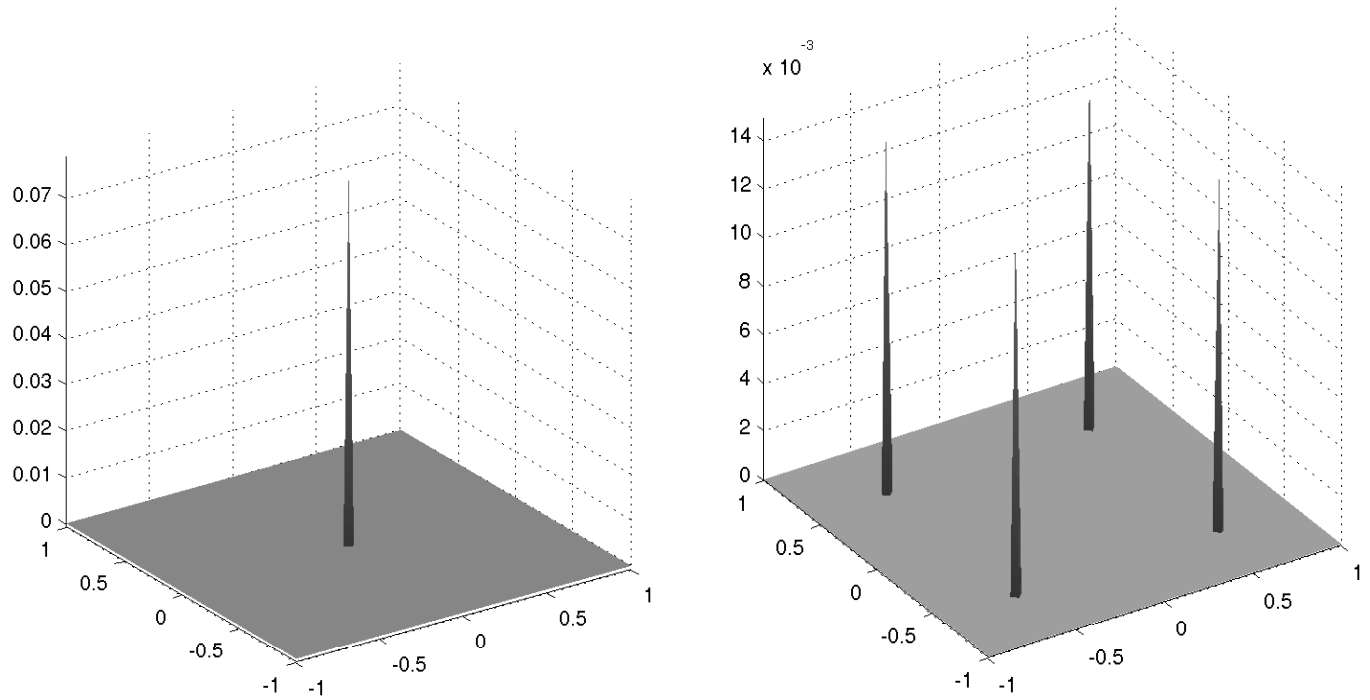

Figure 6. Example 4.2: numerically computed Lagrange multipliers at $h \approx 0.005$.

Therefore, since our arguments are close to the ones of [2,19] or [23], we only briefly sketch the way to derive the error estimate for the convenience of the reader. We underline that our perturbation analysis is inspired by the cited references and is based on standard conclusions. In particular, we were partially guided by the work [2].

We explain our arguments for the problems $(\mathrm{NP})$ and $\left(\mathrm{NP}_{h}\right)$ but they apply to any nonlinear programming problem of this form satisfying the approximation Assumption 5.1 below, with functions $f, f_{h}, G_{i}, G_{h, i}: \mathbb{R}^{m} \rightarrow$ $\mathbb{R}, i=1, \ldots, \ell, h>0$, which are assumed to be of class $C^{2,1}$.

Assumption 5.1. There exist a constant $C>0$ and a function $\alpha: \mathbb{R}_{+} \rightarrow \mathbb{R}_{+}$with $\alpha(h) \rightarrow 0$ as $h \rightarrow 0$ such that

$$
\begin{aligned}
\left|f(u)-f_{h}(w)\right|+\left|f^{\prime}(u)-f_{h}^{\prime}(w)\right|+\left\|f^{\prime \prime}(u)-f_{h}^{\prime \prime}(w)\right\| & +\sum_{i=1}^{\ell}\left(\left|G_{i}(u)-G_{h, i}(w)\right|\right. \\
& \left.+\left|G_{i}^{\prime}(u)-G_{h, i}^{\prime}(w)\right|+\left\|G_{i}^{\prime \prime}(u)-G_{h, i}^{\prime \prime}(w)\right\|\right) \leq C(|u-w|+\alpha(h))
\end{aligned}
$$

holds for all $h>0$ and all $u, w \in \mathcal{U}_{a d}$.

Notice that by Lemmas 3.3 and 3.4, the problems (NP) and $\left(\mathrm{NP}_{h}\right)$ satisfy Assumption 5.1 with $\alpha(h)=$ $h^{2}|\log h|$. To cover the given equality and inequality constraints in a unified way, we introduce the cone $K$ of "nonnegative" vectors by

$$
K=\left\{z \in \mathbb{R}^{\ell}: z_{i}=0, i=1, \ldots k, z_{i} \geq 0, i=k+1, \ldots, \ell\right\} .
$$

Then the constraints $G_{i}(u)=0, i=1, \ldots k, G_{i}(u) \leq 0, i=k+1, \ldots, \ell$, can be expressed by $G(u) \leq_{K} 0$, where $z \leq_{K} 0 \Leftrightarrow-z \in K$. Moreover, we introduce $G(u)=\left[G_{1}(u), \ldots, G_{\ell}(u)\right]^{\top}$ and define $G_{h}$ analogously. By these definitions, $(\mathrm{NP})$ and $\left(\mathrm{NP}_{h}\right)$ can be written as

$$
\text { (P) }\left\{\begin{array} { l } 
{ \operatorname { m i n } f ( u ) } \\
{ G ( u ) \leq _ { K } 0 , \quad u \in \mathcal { U } _ { a d } }
\end{array} \quad \text { and } \quad ( \mathrm { P } _ { h } ) \quad \left\{\begin{array}{l}
\min f_{h}(u) \\
G_{h}(u) \leq_{K} 0, \quad u \in \mathcal{U}_{a d}
\end{array}\right.\right.
$$




\subsection{First- and second-order optimality conditions}

\subsubsection{First-order necessary conditions}

Let us assume once and for all that $\bar{u}$ is a locally optimal solution to (P). According to Robinson [28], we require the following regularity condition at $\bar{u}$ :

$$
0 \in \operatorname{int}\left\{G(\bar{u})+G^{\prime}(\bar{u})\left(\mathcal{U}_{a d}-\bar{u}\right)+K\right\}
$$

where the set in braces is defined as $\cup\left\{G(\bar{u})+G^{\prime}(\bar{u})(u-\bar{u})+k \mid u \in \mathcal{U}_{a d}, k \in K\right\}$. A vector $\bar{u}$ that satisfies all constraints of $(\mathrm{P})$ and the condition (5.1) is said to be regular.

Definition 5.1. The Lagrange function $\mathcal{L}: \mathbb{R}^{m} \times \mathbb{R}^{\ell} \rightarrow \mathbb{R}$ associated with $(\mathrm{P})$ is defined by

$$
\mathcal{L}(u, \mu)=f(u)+\sum_{i=1}^{\ell} \mu_{i} G_{i}(u)
$$

The well known first-order conditions for $(\mathrm{P})$ are stated in the following theorem.

Theorem 5.1. Suppose that $\bar{u}$ is locally optimal for $(\mathrm{P})$ and regular. Then there exists a vector $\bar{\mu} \in \mathbb{R}^{\ell}$ with $\mu_{i} \geqslant 0, i=k+1, \ldots, \ell$, such that the variational inequality

$$
\frac{\partial \mathcal{L}}{\partial u}(\bar{u}, \bar{\mu})(u-\bar{u}) \geq 0 \quad \forall u \in \mathcal{U}_{a d}
$$

is satisfied together with the complementary slackness conditions

$$
\bar{\mu}_{i} G_{i}(\bar{u})=0 \quad \text { for } \quad i=k+1, \ldots, \ell
$$

These relations express the standard necessary first-order conditions of nonlinear programming. The existence of Lagrange multipliers follows from the Robinson regularity condition (5.1). This condition is equivalent to the well known regularity condition by Zowe and Kurcyusz [35] that is sufficient for the existence of Lagrange multipliers (and optimality conditions in qualified form).

Remark 5.1. In the particular case of (NP) that is related to the optimal control problem (OCP), the necessary conditions (5.3)-(5.4) can be expressed in terms of optimal control: we define an adjoint state $\varphi \in W_{0}^{1, \sigma}(\Omega)$, $1 \leq \sigma<n /(n-1)$, as the unique solution of the adjoint equation

$$
\left\{\begin{aligned}
A \varphi+\frac{\partial d}{\partial y}(x, \bar{y}, \bar{u}) \varphi & =\frac{\partial L}{\partial y}(x, \bar{y}, \bar{u})+\sum_{i=1}^{\ell} \bar{\mu}_{i} g_{i}^{\prime}\left(\bar{y}\left(x_{i}\right)\right) \delta_{x_{i}} & & \text { in } \Omega \\
\varphi & =0 & & \text { on } \Gamma .
\end{aligned}\right.
$$

Then the variational inequality (5.3) can be written as

$$
d^{\top}(u-\bar{u}) \geqslant 0 \quad \forall u \in \mathcal{U}_{a d}
$$

where $d \in \mathbb{R}^{m}$ is defined by its components

$$
d_{i}:=\int_{\Omega}\left(\frac{\partial L}{\partial u_{i}}(x, \bar{y}, \bar{u})-\varphi(x) \frac{\partial d}{\partial u_{i}}(x, \bar{y}(x), \bar{u})\right) \mathrm{d} x, \quad i=1, \ldots, m .
$$




\subsubsection{Second-order sufficient condition}

We rely on the following strong second-order sufficient condition used by Robinson [29].

Assumption 5.2. For the pair $(\bar{u}, \bar{\nu})$, it holds

$$
v^{\top} \mathcal{L}^{\prime \prime}(\bar{u}, \bar{\nu}) v>0 \quad \forall v \in \hat{C}_{\bar{u}}, v \neq 0,
$$

where $\hat{C}_{\bar{u}}=\left\{v \in \mathbb{R}^{m} \mid G_{i}^{\prime}(\bar{u}) v=0 \forall i \in\{1, \ldots, k\} \cup\left\{i \in\{k+1, \ldots, \ell+2 m\}: \bar{\nu}_{i}>0\right\}\right\}$.

Here and in the following, $\mathcal{L}^{\prime \prime}(u, \mu)$ denotes the Hessian matrix of $\mathcal{L}$ at $(u, \mu)$ with respect to $u$. Later, we will extend the Lagrangian $\mathcal{L}$ to a function $\hat{\mathcal{L}}$ that considers also the inequalities defining $\mathcal{U}_{\text {ad }}$. Then we have $\mathcal{L}^{\prime \prime}=\hat{\mathcal{L}}^{\prime \prime}$, since the associated $G_{i}$ are linear.

The next theorem is a standard result from nonlinear programming theory, $c f$. [15] or [21].

Theorem 5.2. Let $\bar{u}$ satisfy the constraints of $(\mathrm{P})$, the first-order necessary conditions (5.3)-(5.4), and the second-order sufficient Assumption 5.2. Then there exist real numbers $\omega>0$ and $\varepsilon>0$ such that the quadratic growth condition

$$
f(u)-f(\bar{u}) \geqslant \omega|u-\bar{u}|^{2}
$$

holds for all admissible $u$ with $|u-\bar{u}| \leq \varepsilon$.

\subsection{Perturbation analysis}

To set up the first order-necessary conditions for $\left(\mathrm{P}_{h}\right)$, we introduce the associated Lagrange function $\mathcal{L}_{h}: \mathbb{R}^{m+\ell} \rightarrow \mathbb{R}$ by $\mathcal{L}_{h}(u, \mu)=f_{h}(u)+G_{h}(u)^{\top} \mu$. Then the first-order conditions can be formulated as follows:

If $\bar{u}_{h}$ is locally optimal for $\left(\mathrm{P}_{h}\right)$ and the Robinson regularity condition (5.1) is satisfied at $\bar{u}_{h}$ with $G_{h}\left(\bar{u}_{h}\right)$ and $G_{h}^{\prime}\left(\bar{u}_{h}\right)$ substituted for $G(\bar{u})$ and $G^{\prime}(\bar{u})$, respectively, then there exists a Lagrange multiplier $\bar{\mu}_{h} \in \mathbb{R}^{\ell}$ with $\bar{\mu}_{h, i} \geqslant 0, i=k+1, \ldots, \ell$, such that

$$
\begin{array}{r}
\frac{\partial \mathcal{L}_{h}}{\partial u}\left(\bar{u}_{h}, \bar{\mu}_{h}\right)\left(u-\bar{u}_{h}\right) \geq 0 \quad \forall u \in \mathcal{U}_{a d}, \text { and } \\
\bar{\mu}_{h, i} G_{h, i}(\bar{u})=0 \quad \text { for all } i=1, \ldots, \ell
\end{array}
$$

It is not difficult to show that the Robinson regularity condition for $\left(\mathrm{P}_{h}\right)$ is satisfied at $\bar{u}_{h}$, provided that $\bar{u}_{h}$ is sufficiently close to $\bar{u}$. In this case a Lagrange multiplier associated with $\bar{u}_{h}$ exists for all sufficiently small $h$. However, we have to show the existence of a locally optimal $\bar{u}_{h}$ in a neighborhood of $\bar{u}$ and finally, we want to get an estimate for the difference.

In a first step, one can show by the Robinson implicit function theorem that for every sufficiently small $h$ there is a $u_{h}$ admissible for $\left(\mathrm{P}_{h}\right)$ such that the estimate

$$
\left|\bar{u}-u_{h}\right| \leq C \alpha(h)
$$

holds for all sufficiently small $h>0$ with a constant $C$ not depending on $h$. We do not present the details that are fairly standard.

The next result requires some more effort, although it is to be expected. It shows that $\bar{u}$ can be approximated by locally optimal solutions $\bar{u}_{h}$ of the discretized problem. 
Lemma 5.1. Let the reference solution $\bar{u}$ satisfy the linear independence condition (LICQ). Then, for all given $\rho>0$, the auxiliary problem

$$
\left(\mathrm{P}_{h, \rho}\right) \quad\left\{\begin{array}{l}
\min f_{h}(u) \\
G_{h}(u) \leq_{K} 0, \\
u \in \mathcal{U}_{a d} \cap c l B(\bar{u}, \rho)
\end{array}\right.
$$

is solvable for all sufficiently small $h$. If $\bar{u}_{h}$ is any optimal solution to this problem, then there exists an element $v_{h} \in \mathcal{U}_{a d}$ that is admissible for $(\mathrm{P})$ and satisfies the estimate

$$
\left|\bar{u}_{h}-v_{h}\right| \leq C \alpha(h)
$$

with some constant $C>0$ that is independent of $h$.

We have shown this lemma by techniques used in [1]. To shorten the presentation, we outsourced the proof to the forthcoming paper [34].

If $\rho>0$ is taken sufficiently small, $h \in\left(0, h_{0}\right)$ with some $h_{0}>0$ depending on $\rho$, and $\bar{u}$ is a local solution of (P) satisfying the linear independence constraint qualification (LICQ) and the second-order Assumption 5.2, then all solutions $\bar{u}_{h}$ of the auxiliary problem $\left(\mathrm{P}_{h, \rho}\right)$ belong to $B(\bar{u}, \rho)$. Therefore, they are also locally optimal for the problem $\left(\mathrm{P}_{h}\right)$.

Now, we compare the solution $\bar{u}_{h}$ of $\left(\mathrm{P}_{h, \rho}\right)$ defined in (5.11) with $u_{h}$ that is admissible for $\left(\mathrm{P}_{h, \rho}\right)$ and approximates $\bar{u}$ with the order $\alpha(h)$. We get

$$
f_{h}\left(\bar{u}_{h}\right) \leq f_{h}\left(u_{h}\right) \leq\left|f_{h}\left(u_{h}\right)-f_{h}(\bar{u})\right|+\left|f_{h}(\bar{u})-f(\bar{u})\right|+f(\bar{u}) .
$$

By

$$
\left|f_{h}(\bar{u})-f(\bar{u})\right|+\left|u_{h}-\bar{u}\right|+\left|f_{h}\left(\bar{u}_{h}\right)-f\left(\bar{u}_{h}\right)\right| \leq c \alpha(h)
$$

and by the uniform Lipschitz property of $f_{h}$, we find

$$
f\left(\bar{u}_{h}\right) \leq f(\bar{u})+c_{1} \alpha(h) .
$$

Next, we compare $\bar{u}$ with $v_{h}$ taken from Lemma 5.1 that is admissible for (P) and approximates $\bar{u}_{h}$ with the order $\alpha(h)$. From the quadratic growth condition we obtain

$$
f\left(v_{h}\right) \geq f(\bar{u})+\omega\left|\bar{u}-v_{h}\right|^{2} .
$$

Notice that $v_{h}$ is close enough to $\bar{u}$, if $h$ is sufficiently small. From $\left|\bar{u}_{h}-v_{h}\right| \leq c \alpha(h)$ we deduce

$$
f\left(\bar{u}_{h}\right)+c_{2} \alpha(h) \geq f(\bar{u})+\omega\left|\bar{u}-\bar{u}_{h}\right|^{2} .
$$

Combining the inequalities (5.14)-(5.15), it follows that

$$
f(\bar{u})+c_{1} \alpha(h) \geq f(\bar{u})+\omega\left|\bar{u}-\bar{u}_{h}\right|^{2}-c_{2} \alpha(h)
$$

and hence

$$
\left|\bar{u}-\bar{u}_{h}\right| \leq c \sqrt{\alpha(h)}
$$

This auxiliary estimate is not optimal. We are able to get rid of the square root. Moreover, we are able to show local uniqueness of $\bar{u}_{h}$, i.e. uniqueness of local optima of $\left(\mathrm{P}_{h}\right)$ in $B(\bar{u}, \rho)$, if $\rho$ is small enough. Both tasks can be accomplished by the stability theory for optimality systems written as generalized equations, which we work out in more detail. Let us introduce the necessary notions. 
The dual cone associated with $K$ is given by

$$
K_{+}=\left\{z \in \mathbb{R}^{\ell} \mid z_{i} \geq 0, i=k+1, \ldots, \ell\right\}=\mathbb{R}^{k} \times \mathbb{R}_{+}^{\ell-k}
$$

Moreover, we need the normal cone $\partial \psi_{E}(x)$ to a convex set $E \subset \mathbb{R}^{n}$ at a point $x \in \mathbb{R}^{n}$ defined by

$$
\partial \psi_{E}(x)= \begin{cases}z \in \mathbb{R}^{n} \text { with } z^{\top}(e-x) \leq 0 \quad \forall e \in E, & \text { if } x \in E \\ \emptyset, & \text { if } x \notin E .\end{cases}
$$

By the dual cone $K_{+}$, the complementarity conditions $G(u) \leq_{K} 0, \mu \in K_{+}$, and $G(u)^{\top} \mu=0$ can be compressed to

$$
G(u)^{\top}(\eta-\mu) \leq 0 \quad \forall \eta \in K_{+} .
$$

In terms of the normal cone, this reads $G(u) \in \partial \psi_{K_{+}}(\mu)$. Therefore, we can express the first-order necessary conditions for the problems $(\mathrm{P}),(5.3)-(5.4)$, and $\left(\mathrm{P}_{h}\right),(5.9)-(5.10)$, respectively, in the form of the generalized equations

$$
0 \in F(u, \mu)+\mathcal{T}(u, \mu), \quad 0 \in F_{h}\left(u_{h}, \mu_{h}\right)+\mathcal{T}\left(u_{h}, \mu_{h}\right)
$$

In this setting, $F$ and $F_{h}$ are mappings in $\mathbb{R}^{m} \times \mathbb{R}^{\ell}$ defined by

$$
F(u, \mu)=\left[\begin{array}{c}
\nabla_{u} \mathcal{L}(u, \mu) \\
-G(u)
\end{array}\right], \quad \text { and } \quad F_{h}(u, \mu)=\left[\begin{array}{l}
\nabla_{u} \mathcal{L}_{h}(u, \mu) \\
-G_{h}(u)
\end{array}\right] \text {, }
$$

respectively, and $\mathcal{T}$ is a set-valued mapping defined by $\mathcal{T}(u, \mu)=\partial \psi_{\mathcal{U}_{a d}}(u) \times \partial \psi_{K_{+}}(\mu)$. We recall the definition $\hat{G}(u):=\left(G_{i}(u)\right)_{i=1}^{\ell+2 m}$ and define $\hat{K}=K \times \mathbb{R}_{+}^{2 m}$. Then the optimality system for (P) is equivalent to the conditions

$$
\nabla_{u} \hat{\mathcal{L}}(u, \nu)=0, \quad \hat{G}(u) \leq_{\hat{K}} 0, \nu \geq_{\hat{K}_{+}} 0, \hat{G}(u)^{\top} \nu=0
$$

where $\nu$ belongs to $\mathbb{R}^{\ell+2 m}$. The first $\ell$ components of this extended $\nu$ are equal to those of $\mu$. Given $\mu$, it is easy to find the last $2 m$ components of $\nu$. They are obtained by

$$
\begin{array}{ll}
\nu_{i}=\nabla_{u} \mathcal{L}(u, \mu)_{+}, & i=\ell+1, \ldots, \ell+m, \\
\nu_{i}=\nabla_{u} \mathcal{L}(u, \mu)_{-}, & i=\ell+m+1, \ldots, \ell+2 m,
\end{array}
$$

where $a_{+}=(a+|a|) / 2, a_{-}=(-a+|a|) / 2$. By this choice, the complementarity conditions are satisfied and these Lagrange multipliers are unique, if $u_{a}<u_{b}$. We refer, for instance, to [33], Section 2.

Written in terms of a generalized equation, (5.18) reads

$$
0 \in\left(\begin{array}{c}
\nabla_{u} \hat{\mathcal{L}}(u, \nu) \\
-\hat{G}(u)
\end{array}\right)+\left(\begin{array}{c}
\{0\} \\
\partial \psi_{\hat{K}_{+}}(\nu)
\end{array}\right)=: \hat{\mathcal{F}}(u, \nu)+\hat{\mathcal{T}}(u, \nu) .
$$

We assume that the reader is familiar with the notion of strong regularity of generalized equations, cf. Robinson [29]. In the following, the extended Lagrange multiplier vector $\bar{\nu}$ is defined by $\bar{\mu}$ according to (5.19). It follows from Robinson [29] that the generalized equation (5.20) is strongly regular at $(\bar{u}, \bar{\nu})$, if the linear independence condition (LICQ) and the strong second-order sufficient Assumption 5.2 are fulfilled for $(\bar{u}, \bar{\nu})$. 
In view of this, we can apply the Robinson implicit function theorem [29], Theorem 2.1, to the generalized equation

$$
\delta \in \hat{\mathcal{F}}(u, \nu)+\hat{\mathcal{T}}(u, \nu)
$$

with given perturbation $\delta \in \mathbb{R}^{\ell+2 m}$. In the case $\delta=0$, we know that the pair $(\bar{u}, \bar{\nu})$ is a solution for (5.21).

As a consequence of the implicit function theorem, there are positive values $r, \sigma$ such that, for all $\delta \in B(0, r)$, the generalized equation (5.21) has exactly one solution $(u, \nu)$ in $B((\bar{u}, \bar{\nu}), \sigma)$, i.e. we have local uniqueness. Moreover, there is a constant $C>0$ such that the Lipschitz property

$$
|u-\bar{u}|+|\nu-\bar{\nu}| \leq C|\delta|
$$

is satisfied. By the linearity of the Lagrange function with respect to $\nu$ and by (LICQ), the Lagrange multipliers $\bar{\nu}$ and $\nu$ associated with $\bar{u}$ and $u$, respectively, are unique.

To apply the Robinson implicit function theorem, we need some further results. We recall the well known fact that (LICQ) implies boundedness of the Lagrange multipliers, stated in the following lemma.

Lemma 5.2. Assume that the linear independence condition (LICQ) is satisfied at $\bar{u}$, and that $\bar{u}_{h}$ is a sequence of local solutions to problem $\left(P_{h}\right)$ converging to $\bar{u}$ as $h \downarrow 0$. Then the Lagrange multipliers $\bar{\nu}_{h}$ associated with $\bar{u}_{h}$ are uniformly bounded for all sufficiently small $h>0$.

Next, we consider the solution $\bar{u}_{h}$ of the approximate problem $\left(\mathrm{P}_{h, \rho}\right)$ according to Lemma 5.1. With an associated Lagrange multiplier $\bar{\nu}_{h}$, it satisfies the generalized equation

$$
0 \in \hat{\mathcal{F}}_{h}\left(\bar{u}_{h}, \bar{\nu}_{h}\right)+\hat{\mathcal{T}}\left(\bar{u}_{h}, \bar{\nu}_{h}\right)
$$

This inclusion is unperturbed in its left-hand side but contains the "approximated" function $\hat{\mathcal{F}}_{h}$ depending on $h$. We show that $\left(\bar{u}_{h}, \bar{\nu}_{h}\right)$ solves the perturbed generalized equation

$$
\delta_{h} \in \hat{\mathcal{F}}\left(\bar{u}_{h}, \bar{\nu}_{h}\right)+\hat{\mathcal{T}}\left(\bar{u}_{h}, \bar{\nu}_{h}\right)
$$

with the "exact" function $\hat{\mathcal{F}}$ but a perturbation $\delta_{h}$ of the order $\alpha(h)$.

Lemma 5.3. Let $\left\{\left(\bar{u}_{h}, \bar{\nu}_{h}\right)\right\}$ be a sequence of solutions to the inclusion (5.23). Assume that $\bar{u}_{h} \rightarrow \bar{u}$, as $h \downarrow 0$, where $\bar{u}$ is a local solution to $(\mathrm{P})$ satisfying the linear independence condition (LICQ). Then the pair $\left(\bar{u}_{h}, \bar{\nu}_{h}\right)$ solves the generalized equation (5.24) with a perturbation $\delta_{h}$ of the order $\alpha(h)$.

Proof. The generalized equation (5.23) is the $h$-version of (5.20),

$$
0 \in\left(\begin{array}{ccc}
\nabla_{u} \hat{\mathcal{L}_{h}}(\bar{u}, \bar{\nu}) & + & \{0\} \\
-\hat{G}_{h}(\bar{u}) & + & \partial \psi_{\hat{K}_{+}}(\bar{\nu})
\end{array}\right) .
$$

We consider first the upper equation,

$$
\begin{aligned}
0 & =\nabla_{u} \hat{\mathcal{L}_{h}}\left(\bar{u}_{h}, \bar{\nu}_{h}\right)=\nabla f_{h}\left(\bar{u}_{h}\right)+\sum_{i=1}^{\ell+2 m} \bar{\nu}_{h, i} \nabla G_{h, i}\left(\bar{u}_{h}\right) \\
& =\nabla f\left(\bar{u}_{h}\right)+r_{h, 1}+\sum_{i=1}^{\ell+2 m} \bar{\nu}_{h, i} \nabla G_{i}\left(\bar{u}_{h}\right)+\sum_{i=1}^{\ell+2 m} \bar{\nu}_{h, i}\left(\nabla G_{h, i}\left(\bar{u}_{h}\right)-\nabla G_{i}\left(\bar{u}_{h}\right)\right) \\
& =\nabla f\left(\bar{u}_{h}\right)+\sum_{i=1}^{\ell+2 m} \bar{\nu}_{h, i} \nabla G_{i}\left(\bar{u}_{h}\right)+r_{h, 1}+r_{h, 2} \\
& =\nabla_{u} \hat{\mathcal{L}}_{h}\left(\bar{u}_{h}, \bar{\nu}_{h}\right)-\delta_{h, 1},
\end{aligned}
$$


where $\left|r_{h, 1}\right|+\left|r_{h, 2}\right|+\left|\delta_{h, 1}\right| \leq c \alpha(h)$. The estimation of $r_{h, 2}$ makes use of the boundedness of the multiplier sequence $\bar{\nu}_{h}$ stated in Lemma 5.2. Analogously, we deduce from the lower inclusion

$$
\begin{aligned}
0 & \in-\hat{G}_{h}\left(\bar{u}_{h}\right)+\partial \psi_{\hat{K}_{+}}\left(\bar{\nu}_{h}\right) \\
& \in-\hat{G}\left(\bar{u}_{h}\right)+\left(\hat{G}\left(\bar{u}_{h}\right)-\hat{G}_{h}\left(\bar{u}_{h}\right)\right)+\partial \psi_{\hat{K}_{+}}\left(\bar{\nu}_{h}\right) \\
& \in-\hat{G}\left(\bar{u}_{h}\right)-\delta_{h, 2}+\partial \psi_{\hat{K}_{+}}\left(\bar{\nu}_{h}\right)
\end{aligned}
$$

with $\left|\delta_{2, h}\right| \leq c \alpha(h)$. Collecting both results, we see that (5.24) is verified with perturbation $\delta_{h}^{\top}=\left(\delta_{h, 1}^{\top}, \delta_{h, 2}^{\top}\right)$ of order $\alpha(h)$.

Now we are able to prove our main stability result on nonlinear programming problems.

Theorem 5.3. Let a locally optimal solution $\bar{u}$ for problem $(\mathrm{P})$ satisfy, together with the Lagrange multiplier $\bar{\nu}$, the linear independence condition (LICQ) and the strong second-order sufficient optimality condition (5.7). Then $\bar{u}$ is locally unique and there exist $\rho>0$ and $h_{0}>0$ such that, for all $h \in\left(0, h_{0}\right)$, problem $\left(\mathrm{P}_{h}\right)$ admits in $B(\bar{u}, \rho)$ a unique locally optimal solution $\bar{u}_{h}$. Associated with $\bar{u}_{h}$, there exists a unique associated Lagrange multiplier $\bar{\nu}_{h}$ and a constant $C>0$ not depending on $h$ such that

$$
\left|\bar{u}-\bar{u}_{h}\right|+\left|\bar{\nu}-\bar{\nu}_{h}\right| \leq C \alpha(h)
$$

Proof. The existence of the sequence $\bar{u}_{h}$ follows from Lemma 5.1, based on the assumptions (LICQ) and (5.7). In particular, we have $\bar{u}_{h} \rightarrow \bar{u}$ as $h \rightarrow 0$. The same assumptions permit to apply the Robinson implicit function theorem to the inclusion (5.24). Lemma 5.3 shows that $\delta_{h} \rightarrow 0$ as $h \rightarrow 0$. Therefore, for all sufficiently small $h>0, \delta_{h}$ belongs to a neighborhood $\mathcal{O}$ of 0 and $\left(\bar{u}_{h}, \bar{\nu}_{h}\right)$ is contained in a neighborhood $W_{\varepsilon}$ of $(\bar{u}, \bar{\nu})$ where $(5.21)$ is uniquely solvable and the dependence on $\delta$ is Lipschitz. In $W_{\varepsilon}$, there is a unique element $(\tilde{u}, \tilde{\nu})$ that satisfies the generalized equation

$$
\delta_{h} \in \hat{\mathcal{F}}(\tilde{u}, \tilde{\nu})+\hat{\mathcal{T}}(\tilde{u}, \tilde{\nu}) .
$$

Moreover, we have the estimate $|\bar{u}-\tilde{u}|+|\nu-\tilde{\nu}| \leq C|\delta|$. By uniqueness, $\tilde{u}=\bar{u}_{h}$ and $\tilde{\nu}=\bar{\nu}_{h}$ must hold. The local uniqueness of $\bar{u}$ follows in the same way by the implicit function theorem applied to the inclusion (5.21) with $\delta=0$ at $(\bar{u}, \bar{\nu})$.

Remark 5.2. Extracting the first $\ell$ components of the multipliers $\bar{\nu}$ and $\nu_{h}$, we obtain the error estimate

$$
\left|\bar{u}-\bar{u}_{h}\right|+\left|\bar{\mu}-\bar{\mu}_{h}\right| \leq C \alpha(h)
$$

that includes only the Lagrange multiplier associated with the state constraints. Conversely, given $\mu$ and $\mu_{h}$, estimate (3.13) follows via the formula (5.19).

Acknowledgements. The authors are grateful to W. Alt (Jena) and D. Klatte (Zurich) for their helpful comments and suggestions. Moreover, we thank the referee who draw our attention to the paper [4].

\section{REFERENCES}

[1] E.L. Allgower, K. Böhmer, F.A. Potra and W.C. Rheinboldt, A mesh-independence principle for operator equations and their discretizations. SIAM J. Numer. Anal. 23 (1986) 160-169.

[2] W. Alt, On the approximation of infinite optimization problems with an application to optimal control problems. Appl. Math. Opt. 12 (1984) 15-27.

[3] N. Arada, E. Casas and F. Tröltzsch, Error estimates for the numerical approximation of a semilinear elliptic control problem. Comput. Optim. Appl. 23 (2002) 201-229.

[4] A. Bermúdez, P. Gamallo and R. Rodríguez, Finite element methods in local active control of sound. SIAM J. Control Optim. 43 (2004) 437-465 (electronic).

[5] F. Bonnans and A. Shapiro, Perturbation Analysis of Optimization Problems. Springer-Verlag, New York, USA (2000). 
[6] S.C. Brenner and L.R. Scott, The Mathematical Theory of Finite Element Methods. Springer, New York, USA (1994).

[7] E. Casas, Boundary control of semilinear elliptic equations with pointwise state constraints. SIAM J. Control Optim. 31 (1993) 993-1006.

[8] E. Casas, Error estimates for the numerical approximation of semilinear elliptic control problems with finitely many state contraints. ESAIM: COCV 8 (2002) 345-374.

[9] E. Casas, Using piecewise linear functions in the numerical approximation of semilinear elliptic control problems. Adv. Comput. Math. 26 (2007) 137-153.

[10] E. Casas and M. Mateos, Second order sufficient optimality conditions for semilinear elliptic control problems with finitely many state constraints. SIAM J. Control Optim. 40 (2002) 1431-1454.

[11] E. Casas and M. Mateos, Uniform convergence of the FEM. Applications to state constrained control problems. J. Comput. Appl. Math. 21 (2002) 67-100.

[12] J.C. de los Reyes, P. Merino, J. Rehberg and F. Tröltzsch, Optimality conditions for state-constrained PDE control problems with finite-dimensional control space. Control Cybern. (to appear).

[13] M. Deckelnick and M. Hinze, Convergence of a finite element approximation to a state constrained elliptic control problem. SIAM J. Numer. Anal. 45 (2007) 1937-1953.

[14] M. Deckelnick and M. Hinze, Numerical analysis of a control and state constrained elliptic control problem with piecewise constant control approximations, in Numerical Mathematics and Advanced Applications, Proc. of ENUMATH 2007, Graz, K. Kunisch, G. Of and O. Steinbach Eds., Springer, Berlin-Heidelberg, Germany (2008) 597-604.

[15] A.V. Fiacco and G.P. McCormick, Nonlinear Programming: Sequential Unconstrained Minimization Techniques. J. Wiley and Sons, Inc., New York, USA (1968).

[16] J. Frehse and R. Rannacher, Eine $l^{1}$-Fehlerabschätzung diskreter Grundlösungen in der Methode der finiten Elemente. Bonner Math. Schriften 89 (1976) 92-114.

[17] D. Gilbarg and N.S. Trudinger, Elliptic Partial Differential Equations of Second Order. Springer, Berlin, Germany (1998).

[18] P. Grisvard, Elliptic Problems in Nonsmooth Domains. Pitman, Boston, USA (1985).

[19] D. Klatte, A note on quantitative stability results in nonlinear optimization. Seminarbericht 90, Humboldt-Universität zu Berlin, Sektion Mathematik, Germany (1987).

[20] D. Klatte and B. Kummer, Nonsmooth Equations in Optimization: Regularity, Calculus, Methods and Applications. Kluwer Academic Publishers, Dordrecht, The Netherlands (2002).

[21] D.G. Luenberger, Linear and Nonlinear Programming. Addison Wesley, Reading, Massachusetts, USA (1984).

[22] K. Malanowski, Stability of solutions to convex problems of optimization, Lecture Notes Contr. Inf. Sci. 93, Springer-Verlag, Berlin, Germany (1987).

[23] K. Malanowski, Ch. Büskens and H. Maurer, Convergence of approximations to nonlinear optimal control problems, in Mathematical Programming with Data Perturbations, A.V. Fiacco Ed., Lecture Notes to Pure and Applied Mathematics 195, Marcel Dekker, New York, USA (1998) 253-284.

[24] C. Meyer, Error estimates for the finite-element approximation of an elliptic control problem with pointwise state and control constraints. Contr. Cybern. 37 (2008) 51-85.

[25] C. Meyer, U. Prüfert and F. Tröltzsch, On two numerical methods for state-constrained elliptic control problems. Otim. Meth. Software 22 (2007) 871-899.

[26] R. Rannacher, Zur $l^{\infty}$-Konvergenz linearer finiter Elemente beim Dirichlet-Problem. Math. Z. 149 (1976) 69-77.

[27] R. Rannacher and B. Vexler, A priori error estimates for the finite element discretization of elliptic parameter identification problems with pointwise measurements. SIAM J. Control Optim. 44 (2005) 1844-1863.

[28] S.M. Robinson, Stability theory for systems of inequalities, II: Differentiable nonlinear systems. SIAM J. Numer. Anal. 13 (1976) 497-513.

[29] S.M. Robinson, Strongly regular generalized equations. Math. Oper. Res. 5 (1980) 43-62.

[30] A. Rösch, Error estimates for linear-quadratic control problems with control constraints. Optim. Methods Softw. 21 (2006) $121-134$.

[31] A.H. Schatz and L.B. Wahlbin, Interior maximum norm estimates for finite element methods. Math. Comp. 31 (1977) $414-442$.

[32] A.H. Schatz and L.B. Wahlbin, Interior maximum-norm estimates for finite element methods, part II. Math. Comp. 64 (1995) 907-928.

[33] F. Tröltzsch, Optimale Steuerung partieller Differentialgleichungen - Theorie, Verfahren und Anwendungen. Vieweg, Wiesbaden, Germany (2005).

[34] F. Tröltzsch, On finite element error estimates for optimal control problems with elliptic PDEs, in The Proceedings of the Conference on Large Scale Scientific Computing, Sozopol, Bulgaria, June 4-8, 2009, Lect. Notes in Comp. Sci., SpringerVerlag (to appear).

[35] J. Zowe and S. Kurcyusz, Regularity and stability for the mathematical programming problem in Banach spaces. Appl. Math. Optim. 5 (1979) 49-62. 